\title{
Quasilinear perpendicular diffusion of cosmic rays in weak dynamical turbulence ${ }^{\star}$
}

\author{
A. Shalchi ${ }^{1,2}$ and R. Schlickeiser ${ }^{1}$ \\ ${ }^{1}$ Institut für Theoretische Physik, Lehrstuhl IV: Weltraum- und Astrophysik, Ruhr-Universität Bochum, 44780 Bochum, \\ Germany \\ 2 Now at: Bartol Research Institute, University of Delaware, Newark, DE 19716, USA \\ e-mail: rsch@tp4.ruhr-uni-bochum.de
}

Received 7 October 2003 / Accepted 4 March 2004

\begin{abstract}
The quasilinear calculation of perpendicular diffusion of cosmic ray particles for weak dynamical magnetic turbulence of arbitrary geometry is presented. Starting from the equations of motion, a detailed point-by-point derivation of quasilinear Fokker-Planck coefficients is given. It is shown that, to have diffusive behaviour of the Fokker-Planck coefficients, the existence of a finite correlation time of the magnetic fluctuations is essential. From the perpendicular Fokker-Planck coefficient $D_{\perp}$, the perpendicular spatial diffusion coefficient $\kappa_{\perp}$ and the associated perpendicular mean free path $\lambda_{\perp}$ are calculated for the damping model of dynamical magnetic turbulence and three different turbulence geometries: slab, 2D and composite turbulence. Explicit analytical expressions for the perpendicular transport parameters of electrons and protons are given for realistic heliospheric plasma parameters. By comparing with our previous determination of the parallel transport parameters, the variation of the ratio of mean free paths $\lambda_{\perp} / \lambda_{\|}$with particle rigidity for the three turbulence models is investigated. The comparison of these predictions with future accurate experimental determinations of the ratio of mean free paths will allow conclusions on the nature of interplanetary magnetic turbulence.
\end{abstract}

Key words. ISM: cosmic rays - plasmas - turbulence - diffusion - Sun: particle emission

\section{Introduction}

The knowledge of transport parameters of energetic charged particles in turbulent magnetized cosmic plasmas is a key problem of cosmic ray astrophysics and space physics. Of particular interest is the diffusion tensor for particle transport parallel and perpendicular to the ordered magnetic field which controls e.g. the penetration and modulation of low-energy cosmic rays in the heliosphere, the confinement and escape of galactic cosmic rays from the Galaxy, and the efficiency of diffusive shock acceleration mechanisms. Although the perpendicular diffusion coefficient plays an essential role in these studies, a rigorous theoretical treatment in the quasilinear limit of weak turbulence and for dynamical magnetic turbulence currently is not available in the literature. Available numerical studies (Michalek \& Ostrowski 1998; Giacalone \& Jokipii 1999; Mace et al. 2000; Michalek 2001) are restricted to magnetostatic turbulence.

It is the purpose of the present paper to provide the quasilinear calculation of perpendicular diffusion for weak dynamical magnetic turbulence of arbitrary geometry. Recently (Shalchi \& Schlickeiser 2003 - hereafter referred to SS03) we

Send offprint requests to: A. Shalchi,

e-mail: andreasm4@yahoo.com

* Appendices A-C are only available in electronic form at http://www.edpsciences.org calculated the parallel mean free path of cosmic ray particles in the composite model and the damping model of dynamical magnetic turbulence (Bieber et al. 1994) using the quasilinear theory (QLT) of particle transport. Here, with the same quasilinear approximation we calculate the relevant Fokker-Planck coefficients and transport parameters for perpendicular diffusion. In Sect. 2 we derive and discuss general expressions for the both FokkerPlanck coefficients $D_{X X}$ and $D_{Y Y}$, which control the perpendicular cosmic ray transport. In Sect. 3 we calculate the Fokker-Planck coefficients for three different turbulence geometries: pure slab-, pure 2D- and composite geometry. With these results it is possible to calculate the spatial diffusion coefficient and the mean free path for perpendicular diffusion (Sect. 4). In Sect. 5 we use the general results of Sect. 4 to calculate the perpendicular diffusion coefficient and the perpendicular mean free path for specific heliospheric plasma parameters. Moreover, we calculate the ratio $\lambda_{\perp} / \lambda_{\|}$and compare it with observations.

\section{Quasilinear particle diffusion}

\subsection{Equations of motion}

The starting point for the derivation of the quasilinear perpendicular spatial diffusion coefficient $\kappa_{\perp}$ are the random 
parts of the Lorentz force for the coordinates $X$ and $Y$ of the guiding center (see Schlickeiser 2002 - Eqs. (S-12.1.9d) and (S-12.1.9e)). For purely magnetic fluctuations these read

$g_{X}=\dot{X}=-v \sqrt{1-\mu^{2}} \cos \phi \frac{\delta B_{\|}}{B_{0}}+\frac{\mu v}{\sqrt{2} B_{0}}\left(\delta B_{R}+\delta B_{L}\right)$

$g_{Y}=\dot{Y}=-v \sqrt{1-\mu^{2}} \sin \phi \frac{\delta B_{\|}}{B_{0}}+\frac{i \mu v}{\sqrt{2} B_{0}}\left(\delta B_{R}-\delta B_{L}\right)$.

In these both equations we used the pitch-angle cosine $\mu$, the particle speed $v$, the gyrophase $\phi$, the magnetic background field $B_{0}$ and the turbulent fields in helical coordinates $\delta B_{R}$ and $\delta B_{L}$. These random force terms determine the corresponding Fokker-Planck coefficients (see Hall \& Sturrock 1968)

$D_{X X}=\lim _{t \rightarrow \infty} \frac{\left\langle(\Delta X)^{2}\right\rangle}{2 t}$,
$D_{Y Y}=\lim _{t \rightarrow \infty} \frac{\left\langle(\Delta Y)^{2}\right\rangle}{2 t}$

which have to be calculated from the ensemble-averaged firstorder corrections to the particle orbits in the weakly turbulent magnetic field. In this section, we go through this derivation point by point. We explicitly calculate the Fokker-Planck coefficient $D_{X X}$; the coefficient $D_{Y Y}$ is calculated in analogous way: we will give the final result but leave the details as exercise to the interested reader.

The perpendicular spatial diffusion coefficient $\kappa_{\perp}$ and the corresponding perpendicular mean free path $\lambda_{\perp}$ are given by the $\mu$-average (see Schlickeiser 2002)

$$
\begin{aligned}
\kappa_{\perp} & =\frac{v \lambda_{\perp}}{3}=\kappa_{X X}+\kappa_{Y Y} \\
& =\frac{1}{2} \int_{-1}^{+1} \mathrm{~d} \mu\left[D_{X X}(\mu)+D_{Y Y}(\mu)\right] \\
& =\frac{1}{2} \int_{-1}^{+1} \mathrm{~d} \mu D_{\perp}(\mu)
\end{aligned}
$$

\subsection{Step 1: Quasilinear approximation}

The quasilinear approximation is achieved by replacing in the Fourier transform of the fluctuating magnetic field

$$
\begin{aligned}
\delta \boldsymbol{B}(\boldsymbol{x}(t), t) & =\int_{-\infty}^{+\infty} \mathrm{d}^{3} k \delta \boldsymbol{b}(\boldsymbol{k}, t) \exp (\iota \boldsymbol{k} \cdot \boldsymbol{x}(t)) \\
& \simeq \int_{-\infty}^{+\infty} \mathrm{d}^{3} k \delta \boldsymbol{b}(\boldsymbol{k}, t) \exp \left({ }_{l} \boldsymbol{k} \cdot \boldsymbol{x}^{0}(t)\right)
\end{aligned}
$$

the true particle orbit $x(t)$ by the unperturbed orbit $x^{0}(t)$, resulting in

$$
\phi(t)=\phi_{0}-\Omega t
$$

and (see Eq. (S-12.2.3a))

$$
\begin{aligned}
\delta B_{L, R, \|} \approx & \sum_{n=-\infty}^{+\infty} \int_{-\infty}^{+\infty} \mathrm{d}^{3} k \delta b_{L, R, \|}(\boldsymbol{k}, t) J_{n}(W) \\
& \times \mathrm{e}^{l n\left(\psi-\phi_{0}\right)+l\left(k_{\|} v_{\|}+n \Omega\right) t+l \boldsymbol{k} \cdot \boldsymbol{x}_{0}},
\end{aligned}
$$

respectively, where $\boldsymbol{x}_{0}=\left(x_{0}, y_{0}, z_{0}\right)$ denotes the initial $(t=0)$ position of the cosmic ray particle. In the last both equations we used the gyrofrequency $\Omega$ and the parameter $W=$ $v / \Omega \cdot k_{\perp} \sqrt{1-\mu^{2}}$. For the wavevector $\boldsymbol{k}$ we used cylindrical coordinates:

$k_{\|}=k_{z}$

$k_{\perp}=\sqrt{k_{x}^{2}+k_{y}^{2}}$

$\psi=\operatorname{arccot}\left(k_{x} / k_{y}\right)$

With these approximations the equation of motion (1) becomes

$$
\begin{aligned}
\frac{\mathrm{d} X}{\mathrm{~d} t} \simeq & -\frac{v \sqrt{1-\mu^{2}}}{B_{0}} \sum_{n=-\infty}^{\infty} \cos \left(\phi_{0}-\Omega t\right) \int \mathrm{d}^{3} k \delta b_{\|}(\boldsymbol{k}, t) \\
& \times J_{n}(W) \mathrm{e}^{\imath \boldsymbol{k} \cdot \boldsymbol{x}_{0}+\ln \left(\psi-\phi_{o}\right)+\imath \beta_{n} t} \\
& +\frac{\mu v}{\sqrt{2} B_{0}} \sum_{n=-\infty}^{\infty} \int \mathrm{d}^{3} k\left[\delta b_{R}(\boldsymbol{k}, t)+\delta b_{R}(\boldsymbol{k}, t)\right] \\
& \times J_{n}(W) \mathrm{e}^{\imath \boldsymbol{k} \cdot \boldsymbol{x}_{0}+\ln \left(\psi-\phi_{o}\right)+\imath \beta_{n} t}
\end{aligned}
$$

where

$\beta_{n} \equiv k_{\|} v_{\|}+n \Omega$

\subsection{Step 2: Formal integration of equation of motion and square of displacement}

If we integrate Eq. (9) over time we obtain with the initial condition $X(t=0)=X_{0}$ for the displacement $\Delta X=X(t)-X_{0}$ :

$$
\begin{aligned}
\Delta X(t)= & -\frac{v \sqrt{1-\mu^{2}}}{B_{0}} \sum_{n=-\infty}^{\infty} \int_{0}^{t} \mathrm{~d} t^{\prime} \cos \left(\phi_{0}-\Omega t^{\prime}\right) \\
& \times \int \mathrm{d}^{3} k \delta b_{\|}\left(\boldsymbol{k}, t^{\prime}\right) J_{n}(W) \mathrm{e}^{\imath \boldsymbol{k} \cdot x_{0}+\imath \beta_{n} t^{\prime}} \\
& \times \mathrm{e}^{i n\left(\psi-\phi_{0}\right)-i m\left(\psi^{\prime}-\phi_{0}\right)} \\
& +\frac{\mu v}{\sqrt{2} B_{0}} \sum_{n=-\infty}^{\infty} \int_{0}^{t} \mathrm{~d} t^{\prime} \\
& \times \int \mathrm{d}^{3} k\left[\delta b_{R}\left(\boldsymbol{k}, t^{\prime}\right)+\delta b_{L}\left(\boldsymbol{k}, t^{\prime}\right)\right] \\
& \times J_{n}(W) \mathrm{e}^{l \boldsymbol{k} \cdot x_{0}+\imath \beta_{n} t^{\prime}} \mathrm{e}^{i n\left(\psi-\phi_{0}\right)-i m\left(\psi^{\prime}-\phi_{0}\right)} .
\end{aligned}
$$


Upon multiplying Eq. (11) with its complex conjugate we find for the square of the displacement

$$
\begin{aligned}
(\Delta X)^{2}= & \frac{v^{2}}{B_{0}^{2}} \sum_{n=-\infty}^{\infty} \sum_{m=-\infty}^{\infty}\left\{\left(1-\mu^{2}\right) \int_{0}^{t} \mathrm{~d} t^{\prime} \int_{0}^{t} \mathrm{~d} t^{\prime \prime} \int \mathrm{d}^{3} k \int \mathrm{d}^{3} k^{\prime}\right. \\
& \times \mathrm{e}^{i n\left(\psi-\phi_{0}\right)-\imath m\left(\psi^{\prime}-\phi_{0}\right)} \cos \left(\phi_{0}-\Omega t^{\prime}\right) \cos \left(\phi_{0}-\Omega t^{\prime \prime}\right) \\
& \times \delta b_{\|}\left(\boldsymbol{k}, t^{\prime}\right) \delta b_{\|}^{*}\left(\boldsymbol{k}^{\prime}, t^{\prime \prime}\right) J_{n}(W) J_{m}\left(W^{\prime}\right) \\
& \times \mathrm{e}^{l\left(\boldsymbol{k}-\boldsymbol{k}^{\prime}\right) \cdot \boldsymbol{x}_{0}+\imath\left(\beta_{n} t^{\prime}-\beta_{m} t^{\prime \prime}\right)} \\
& +\frac{\mu^{2}}{2} \int_{0}^{t} \mathrm{~d} t^{\prime} \int_{0}^{t} \mathrm{~d} t^{\prime \prime} \int \mathrm{d}^{3} k \int \mathrm{d}^{3} k^{\prime} \\
& \times \mathrm{e}^{i n\left(\psi-\phi_{0}\right)-\imath m\left(\psi^{\prime}-\phi_{0}\right)} \\
& \times\left(\delta b_{R}\left(\boldsymbol{k}, t^{\prime}\right)+\delta b_{L}\left(\boldsymbol{k}, t^{\prime}\right)\right)\left(\delta b_{R}^{*}\left(\boldsymbol{k}^{\prime}, t^{\prime \prime}\right)+\delta b_{L}^{*}\left(\boldsymbol{k}^{\prime}, t^{\prime \prime}\right)\right) \\
& \times J_{n}(W) J_{m}\left(W^{\prime}\right) \mathrm{e}^{l\left(\boldsymbol{k}-\boldsymbol{k}^{\prime}\right) \cdot \boldsymbol{x}_{0}+\imath\left(\beta_{n} t^{\prime}-\beta_{m} t^{\prime \prime}\right)} \\
& -\frac{\mu \sqrt{1-\mu^{2}}}{\sqrt{2}} \int_{0}^{t} \mathrm{~d} t^{\prime} \int_{0}^{t} \mathrm{~d} t^{\prime \prime} \int \mathrm{d}^{3} k \int \mathrm{d}^{3} k^{\prime} \\
& \times \delta b_{\|}\left(\boldsymbol{k}, t^{\prime}\right)\left[\delta b_{R}^{*}\left(\boldsymbol{k}^{\prime}, t^{\prime \prime}\right)+\delta b_{L}^{*}\left(\boldsymbol{k}^{\prime}, t^{\prime \prime}\right)\right] \\
&
\end{aligned}
$$$$
\times J_{n}(W) J_{m}\left(W^{\prime}\right) \mathrm{e}^{\imath\left(\beta_{n} t^{\prime}-\beta_{m} t^{\prime \prime}\right)}
$$$$
-\frac{\mu \sqrt{1-\mu^{2}}}{\sqrt{2}} \int_{0}^{t} \mathrm{~d} t^{\prime} \int_{0}^{t} \mathrm{~d} t^{\prime \prime} \int \mathrm{d}^{3} k \int \mathrm{d}^{3} k^{\prime}
$$$$
\times \mathrm{e}^{i n\left(\psi-\phi_{0}\right)-\imath m\left(\psi^{\prime}-\phi_{0}\right)} \cos \left(\phi_{0}-\Omega t^{\prime \prime}\right)
$$$$
\times\left[\delta b_{R}\left(\boldsymbol{k}, t^{\prime}\right)+\delta b_{L}\left(\boldsymbol{k}, t^{\prime}\right)\right] \delta b_{\|}^{*}\left(\boldsymbol{k}^{\prime}, t^{\prime \prime}\right) J_{n}(W) J_{m}(W)
$$$$
\left.\times \mathrm{e}^{l\left(k-k^{\prime}\right) \cdot x_{0}+l\left(\beta_{n} t^{\prime}-\beta_{m} t^{\prime \prime}\right)}\right\}
$$

\subsection{Step 3: Homogeneous turbulence}

Now we use that the turbulence fields are homogenously distributed, and average Eq. (12) over the initial spatial position of the cosmic ray particles using

$\frac{1}{(2 \pi)^{3}} \int_{-\infty}^{+\infty} \mathrm{d}^{3} x_{0} \mathrm{e}^{l\left(\boldsymbol{k}-\boldsymbol{k}^{\prime}\right) \cdot \boldsymbol{x}_{0}}=\delta\left(\boldsymbol{k}-\boldsymbol{k}^{\prime}\right)$

implying that turbulence fields at different wavevectors are uncorrelated. The respective average of Eq. (12) with

$\left\langle\delta b_{l}\left(\boldsymbol{k}, t^{\prime}\right) \delta b_{m}^{*}\left(\boldsymbol{k}^{\prime}, t^{\prime \prime}\right)\right\rangle=\delta\left(\boldsymbol{k}-\boldsymbol{k}^{\prime}\right) P_{l m}\left(\boldsymbol{k}, t^{\prime}-t^{\prime \prime}\right)$ then yields after performing the $\boldsymbol{k}^{\prime}$-integration

$$
\begin{aligned}
&\left\langle(\Delta X)^{2}\right\rangle= \frac{v^{2}}{B_{0}^{2}} \sum_{n=-\infty}^{\infty} \sum_{m=-\infty}^{\infty}\left\{\left(1-\mu^{2}\right) \int_{0}^{t} \mathrm{~d} t^{\prime} \int_{0}^{t} \mathrm{~d} t^{\prime \prime} \int \mathrm{d}^{3} k\right. \\
& \times \mathrm{e}^{l(n-m)\left(\psi-\phi_{0}\right)} \cos \left(\phi_{0}-\Omega t^{\prime}\right) \\
& \times \cos \left(\phi_{0}-\Omega t^{\prime \prime}\right) P_{|\||}\left(\boldsymbol{k}, t^{\prime}-t^{\prime \prime}\right) \\
& \times J_{n}(W) J_{m}(W) \mathrm{e}^{l\left(\beta_{n} t^{\prime}-\beta_{m} t^{\prime \prime}\right)} \\
&+\frac{\mu^{2}}{2} \int_{0}^{t} \mathrm{~d} t^{\prime} \int_{0}^{t} \mathrm{~d} t^{\prime \prime} \int \mathrm{d}^{3} k \mathrm{e}^{l(n-m)\left(\psi-\phi_{0}\right)} \\
& \times\left[P_{R R}\left(\boldsymbol{k}, t^{\prime}-t^{\prime \prime}\right)+P_{R L}\left(\boldsymbol{k}, t^{\prime}-t^{\prime \prime}\right)\right. \\
&\left.+P_{L R}\left(\boldsymbol{k}, t^{\prime}-t^{\prime \prime}\right)+P_{L L}\left(\boldsymbol{k}, t^{\prime}-t^{\prime \prime}\right)\right] \\
& \times J_{n}(W) J_{m}(W) \mathrm{e}^{\imath\left(\beta_{n} t^{\prime}-\beta_{m} t^{\prime \prime}\right)} \\
&-\frac{\mu \sqrt{1-\mu^{2}}}{\sqrt{2}} \int_{0}^{t} \mathrm{~d} t^{\prime} \int_{0}^{t} \mathrm{~d} t^{\prime \prime} \int \mathrm{d}^{3} k \\
& \times \mathrm{e}^{l(n-m)\left(\psi-\phi_{0}\right)} \cos \left(\phi_{0}-\Omega t^{\prime}\right) \\
& \times\left[P_{\| R}\left(\boldsymbol{k}, t^{\prime}-t^{\prime \prime}\right)+P_{\| L}\left(\boldsymbol{k}, t^{\prime}-t^{\prime \prime}\right)\right] \\
& \times J_{n}(W) J_{m}(W) \mathrm{e}^{\imath\left(\beta_{n} t^{\prime}-\beta_{m} t^{\prime \prime}\right)} \\
&-\frac{\mu \sqrt{1-\mu^{2}}}{\sqrt{2}} \int_{0}^{t} \mathrm{~d} t^{\prime} \int_{0}^{t} \mathrm{~d} t^{\prime \prime} \int \mathrm{d}^{3} k \cos \left(\phi_{0}-\Omega t^{\prime \prime}\right) \\
& \times\left[P_{R \|}\left(\boldsymbol{k}, t^{\prime}-t^{\prime \prime}\right)+P_{L \|}\left(\boldsymbol{k}, t^{\prime}-t^{\prime \prime}\right)\right] \\
&\left.\times J_{n}(W) J_{m}(W) \mathrm{e}^{\imath\left(\beta_{n} t^{\prime}-\beta_{m} t^{\prime \prime}\right)}\right\} \\
&
\end{aligned}
$$

\subsection{Step 4: Random phase approximation}

Next we assume that the initial phase $\phi_{0}$ of the cosmic ray particle is a random variable that can take on any value between 0 and $2 \pi$. The averaging of Eq. (15) over $\phi_{0}$ then represents exactly the ensemble-averaging over the turbulent magnetic field. Using

$\frac{1}{2 \pi} \int_{0}^{2 \pi} \mathrm{d} \phi_{0} \mathrm{e}^{\left(l(n-m) \phi_{0}\right)}=\delta_{n, m}$

the double sum over $n$ and $m$ in Eq. (16) is reduced to a single sum. We obtain after some straightforward resumming

$$
\begin{aligned}
& \frac{1}{2 \pi} \int_{0}^{2 \pi} \mathrm{d} \phi_{0}\left\langle(\Delta X)^{2}\right\rangle=\frac{v^{2}}{B_{0}^{2}} \sum_{n=-\infty}^{\infty} \int \mathrm{d}^{3} k \int_{0}^{t} \mathrm{~d} t^{\prime} \int_{0}^{t} \mathrm{~d} t^{\prime \prime} \mathrm{e}^{\imath \beta_{n}\left(t^{\prime}-t^{\prime \prime}\right)} \\
& \quad \times\left\{\frac { 1 } { 4 } ( 1 - \mu ^ { 2 } ) P _ { \| \| \| } ( \boldsymbol { k } , t ^ { \prime } - t ^ { \prime \prime } ) \left[J_{n+1}^{2}(W)+J_{n-1}^{2}(W)\right.\right. \\
&\left.+J_{n+1}(W) J_{n-1}(W)\left(\mathrm{e}^{2 \imath \psi}+\mathrm{e}^{-2 \imath \psi}\right)\right] \\
&+\frac{\mu^{2}}{2} J_{n}^{2}(W)\left[P_{R R}\left(\boldsymbol{k}, t^{\prime}-t^{\prime \prime}\right)+P_{R L}\left(\boldsymbol{k}, t^{\prime}-t^{\prime \prime}\right)\right. \\
&\left.+P_{L R}\left(\boldsymbol{k}, t^{\prime}-t^{\prime \prime}\right)+P_{L L}\left(\boldsymbol{k}, t^{\prime}-t^{\prime \prime}\right)\right] \\
&-\frac{\mu \sqrt{1-\mu^{2}}}{2 \sqrt{2}} J_{n}(W)\left[\left(P_{L \|}\left(\boldsymbol{k}, t^{\prime}-t^{\prime \prime}\right)+P_{R \|}\left(\boldsymbol{k}, t^{\prime}-t^{\prime \prime}\right)\right)\right. \\
& \times\left(J_{n+1}(W) \mathrm{e}^{-\imath \psi}+J_{n-1}(W) \mathrm{e}^{+\imath \psi}\right) \\
&+\left(P_{\| L}\left(\boldsymbol{k}, t^{\prime}-t^{\prime \prime}\right)+P_{\| R}\left(\boldsymbol{k}, t^{\prime}-t^{\prime \prime}\right)\right) \\
&\left.\times\left(J_{n+1}(W) \mathrm{e}^{\imath \psi}+J_{n-1}(W) \mathrm{e}^{-\imath \psi}\right]\right\}
\end{aligned}
$$




\subsection{Step 5: Temporal behaviour of magnetic field correlation tensor}

To proceed we have to specify the time behaviour of the magnetic field correlation tensor $P_{l m}\left(\boldsymbol{k}, t^{\prime}-t^{\prime \prime}\right)$. We assume that all tensor components have the same temporal behaviour, i.e.

$P_{l m}\left(\boldsymbol{k}, t^{\prime}-t^{\prime \prime}\right)=P_{l m}^{0}(\boldsymbol{k}) F\left(\boldsymbol{k}, t^{\prime}-t^{\prime \prime}\right)$.

This assumption allows us to disentangle the time and $\boldsymbol{k}$-integrations in Eq. (17). We find

$$
\begin{aligned}
& \frac{1}{2 \pi} \int_{0}^{2 \pi} \mathrm{d} \phi_{0}\left\langle(\Delta X)^{2}\right\rangle=\frac{v^{2}}{B_{0}^{2}} \sum_{n=-\infty}^{\infty} \int \mathrm{d}^{3} k T\left(t, \beta_{n}\right) \\
& \quad \times\left\{\frac { 1 } { 4 } ( 1 - \mu ^ { 2 } ) P _ { \| \| | } ^ { 0 } ( \boldsymbol { k } ) \left[J_{n+1}^{2}(W)+J_{n-1}^{2}(W)\right.\right. \\
&\left.+J_{n+1}(W) J_{n-1}(W)\left(\mathrm{e}^{2 \imath \psi}+\mathrm{e}^{-2 \imath \psi}\right)\right] \\
&+\frac{\mu^{2}}{2} J_{n}^{2}(W)\left(P_{R R}^{0}(\boldsymbol{k})+P_{R L}^{0}(\boldsymbol{k})+P_{L R}^{0}(\boldsymbol{k})+P_{L L}^{0}(\boldsymbol{k})\right) \\
&-\frac{\mu \sqrt{1-\mu^{2}}}{2 \sqrt{2}} J_{n}(W)\left[( P _ { L \| } ^ { 0 } ( \boldsymbol { k } ) + P _ { R \| \| } ^ { 0 } ( \boldsymbol { k } ) ) \left(J_{n+1}(W) \mathrm{e}^{-\imath \psi}\right.\right. \\
&\left.+J_{n-1}(W) \mathrm{e}^{\imath \psi}\right)\left(P_{\| L}^{0}(\boldsymbol{k})+P_{\| R}^{0}(\boldsymbol{k})\right) \\
&\left.\left.\times\left(J_{n+1}(W) \mathrm{e}^{\imath \psi}+J_{n-1}(W) \mathrm{e}^{-\imath \psi}\right)\right]\right\}
\end{aligned}
$$

where we defined the so-called resonance function

$T\left(t, \beta_{n}\right) \equiv \int_{0}^{t} \mathrm{~d} t^{\prime} \int_{0}^{t} \mathrm{~d} t^{\prime \prime} \mathrm{e}^{\imath \beta_{n}\left(t^{\prime}-t^{\prime \prime}\right)} F\left(\boldsymbol{k}, t^{\prime}-t^{\prime \prime}\right)$.

The behaviour of the function $T$ for large times determines whether perpendicular cosmic ray transport is diffusive (i.e. $\lim _{t \rightarrow \infty} T \propto t$ ), subdiffusive $\left(\lim _{t \rightarrow \infty} T \propto t^{s}, s<1\right.$ ), or superdiffusive (i.e. $\lim _{t \rightarrow \infty} T \propto t^{s}, s>1$ ), respectively.

\subsection{Step 6: Conditions for diffusive perpendicular transport}

We demonstrate here that diffusive perpendicular transport always exists under two conditions:

(a) the time correlation function $F$ of magnetic turbulence depends only on the absolute value of the difference $t^{\prime}-t^{\prime \prime}$, i.e. $F\left(\boldsymbol{k}, t^{\prime}-t^{\prime \prime}\right)=F\left(\boldsymbol{k},\left|t^{\prime}-t^{\prime \prime}\right|\right)$;

(b) there exists a finite correlation time $t_{\mathrm{c}}(\boldsymbol{k})$, that can be wavenumber dependent, beyond which the correlation function $F$ falls to a negligible magnitude.

As one particular choice of the correlation function $F$ we consider here the exponential function

$$
\begin{aligned}
F\left(\boldsymbol{k},\left|t^{\prime}-t^{\prime \prime}\right|\right) & =\exp \left(-\eta(\boldsymbol{k})\left|t^{\prime}-t^{\prime \prime}\right|\right), \\
t_{\mathrm{c}}(\boldsymbol{k}) & =\eta^{-1}(\boldsymbol{k}) .
\end{aligned}
$$

This choice is justified in case of plasma wave turbulence (Schlickeiser 2002, Sect. 12.2.2) where $\eta$ then has to be identified with the wave damping rate. Another choice in accord with the general requirements (a) and (b) would be the damping model of dynamical magnetic turbulence and the random sweeping turbulence, discussed by Bieber et al. (1994).

\subsection{Step 7: Resonance function for the exponential correlation function (21)}

With Eq. (21) inserted into Eq. (20) we obtain

$T\left(t, \beta_{n}, \eta\right)=\int_{0}^{t} \mathrm{~d} t^{\prime} \int_{0}^{t} \mathrm{~d} t^{\prime \prime} \mathrm{e}^{\imath \beta_{n}\left(t^{\prime}-t^{\prime \prime}\right)-\eta\left|t^{\prime}-t^{\prime \prime}\right|}$.

The $t^{\prime \prime}$-integration is now split into the two intervals

(i) $0 \leq t^{\prime \prime} \leq t^{\prime}$ where $\left|t^{\prime}-t^{\prime \prime}\right|=t^{\prime}-t^{\prime \prime}$,

and

(ii) $t^{\prime} \leq t^{\prime \prime} \leq t$ where $\left|t^{\prime}-t^{\prime \prime}\right|=t^{\prime \prime}-t^{\prime}$,

so that

$$
\begin{aligned}
T\left(t, \beta_{n}, \eta\right)= & \int_{0}^{t} \mathrm{~d} t^{\prime}\left[\int_{0}^{t^{\prime}} \mathrm{d} t^{\prime \prime} \mathrm{e}^{\imath \beta_{n}\left(t^{\prime}-t^{\prime \prime}\right)-\eta\left(t^{\prime}-t^{\prime \prime}\right)}\right. \\
& \left.+\int_{t^{\prime}}^{t} \mathrm{~d} t^{\prime \prime} \mathrm{e}^{\imath \beta_{n}\left(t^{\prime}-t^{\prime \prime}\right)-\eta\left(t^{\prime \prime}-t^{\prime}\right)}\right] .
\end{aligned}
$$

After changing integration variables to $s_{1}=t^{\prime}-t^{\prime \prime}$ and $s_{2}=$ $t^{\prime \prime}-t^{\prime}$ in the first and second $t^{\prime \prime}$-integral, respectively, we obtain after straightforward algebra

$$
\begin{aligned}
& T\left(t, \beta_{n}, \eta\right)=\int_{0}^{t} \mathrm{~d} t^{\prime}\left[\int_{0}^{t^{\prime}} \mathrm{d} s_{1} \mathrm{e}^{-\left(\eta-\imath \beta_{n}\right) s_{1}}+\int_{0}^{t-t^{\prime}} \mathrm{d} s_{2} \mathrm{e}^{-\left(\eta+\imath \beta_{n}\right) s_{2}}\right] \\
& =\int_{0}^{t} \mathrm{~d} t^{\prime}\left[\frac{1-\mathrm{e}^{-\left(\eta-\imath \beta_{n}\right) t^{\prime}}}{\eta-\imath \beta_{n}}+\frac{1-\mathrm{e}^{-\left(\eta+\imath \beta_{n}\right)\left(t-t^{\prime}\right)}}{\eta+\imath \beta_{n}}\right] \\
& =\frac{1}{\eta-\imath \beta_{n}}\left[t-\frac{1-\mathrm{e}^{-\left(\eta-\imath \beta_{n}\right) t}}{\eta-\imath \beta_{n}}\right]+\frac{t}{\eta+\imath \beta_{n}} \\
& -\frac{\mathrm{e}^{-\left(\eta+\imath \beta_{n}\right) t}}{\eta+\imath \beta_{n}} \int_{0}^{t} \mathrm{~d} t^{\prime} \mathrm{e}^{\left(\eta+\imath \beta_{n}\right) t^{\prime}} \\
& =\frac{2 \eta t}{\eta^{2}+\beta_{n}^{2}}+\frac{\mathrm{e}^{-\left(\eta-\imath \beta_{n}\right) t}-1}{\left(\eta-\imath \beta_{n}\right)^{2}}+\frac{\mathrm{e}^{-\left(\eta+\imath \beta_{n}\right) t}-1}{\left(\eta+\imath \beta_{n}\right)^{2}} \\
& =\frac{2 \eta}{\eta^{2}+\beta_{n}^{2}}\left[t-\frac{2 \beta_{n} \mathrm{e}^{-\eta t} \sin \beta_{n} t}{\eta^{2}+\beta_{n}^{2}}\right] \\
& -\frac{2\left(\eta^{2}-\beta_{n}^{2}\right)}{\left(\eta^{2}+\beta_{n}^{2}\right)^{2}}\left[1-\mathrm{e}^{-\eta t} \cos \beta_{n} t\right] \text {. }
\end{aligned}
$$

\subsubsection{Fokker-Planck coefficient $D_{\mathrm{XX}}$}

First of all, we note that for times much larger than the correlation time, $t \gg \eta^{-1}=t_{\mathrm{c}}$, the resonance function (24) approaches the limit

$T\left(t \gg t_{\mathrm{c}}\right) \rightarrow \frac{2 \eta t}{\eta^{2}+\beta_{n}^{2}}$,

proving that the transport indeed is diffusive for large times. According to the definition (3) we have to take the limit

$\lim _{t \rightarrow \infty} \frac{T\left(t \gg t_{\mathrm{c}}\right)}{2 t}=\mathcal{R}(\boldsymbol{k})=\frac{\eta}{\eta^{2}+\beta_{n}^{2}}$. 
With Eq. (19) the Fokker-Planck coefficient $D_{X X}$ then becomes

$$
\begin{aligned}
D_{X X}= & \lim _{t \rightarrow \infty} \frac{1}{2 t} \frac{1}{2 \pi} \int_{0}^{2 \pi} \mathrm{d} \phi_{0}\left\langle(\Delta X)^{2}\right\rangle \\
= & \frac{v^{2}}{B_{0}^{2}} \sum_{n=-\infty}^{\infty} \int \mathrm{d}^{3} k \mathcal{R}(\boldsymbol{k}) \\
& \times\left\{\frac { 1 } { 4 } ( 1 - \mu ^ { 2 } ) P _ { | \| | | } ^ { 0 } ( \boldsymbol { k } ) \left[J_{n+1}^{2}(W)+J_{n-1}^{2}(W)\right.\right. \\
& \left.+J_{n+1}(W) J_{n-1}(W)\left(\mathrm{e}^{2 \imath \psi}+\mathrm{e}^{-2 u}\right)\right] \\
& +\frac{\mu^{2}}{2} J_{n}^{2}(W)\left(P_{R R}^{0}(\boldsymbol{k})+P_{R L}^{0}(\boldsymbol{k})+P_{L R}^{0}(\boldsymbol{k})+P_{L L}^{0}(\boldsymbol{k})\right) \\
& -\frac{\mu \sqrt{1-\mu^{2}}}{2 \sqrt{2}} J_{n}(W)\left[( P _ { L \| } ^ { 0 } ( \boldsymbol { k } ) + P _ { R \| } ^ { 0 } ( \boldsymbol { k } ) ) \left(J_{n+1}(W) \mathrm{e}^{-\imath \psi}\right.\right. \\
& \left.+J_{n-1}(W) \mathrm{e}^{+\imath \psi}\right)+\left(P_{\| L}^{0}(\boldsymbol{k})+P_{\| R}^{0}(\boldsymbol{k})\right)\left(J_{n+1}(W) \mathrm{e}^{\imath \psi}\right. \\
& \left.\left.\left.+J_{n-1}(W) \mathrm{e}^{-\imath \psi}\right)\right]\right\} .
\end{aligned}
$$

This completes the derivation of the Fokker-Planck coefficient $D_{X X}$ for general turbulence geometries. For further reduction, the turbulence geometry has to be specified via the tensor $P_{l m}^{0}(\boldsymbol{k})$. This will be the subject of the next sections.

\subsubsection{Special cases}

It is instructive to inspect several special cases of the resonance function (24). For an infinitely large correlation time $t_{\mathrm{c}}=\infty$, corresponding to $\eta=0$, Eq. (24) reduces to

$T(\eta=0)=\frac{2\left[1-\cos \beta_{n} t\right]}{\beta_{n}^{2}}=\frac{4 \sin ^{2}\left(\beta_{n} t / 2\right)}{\beta_{n}^{2}}$.

As has been noted before by Jaekel \& Schlickeiser (1992) for large $t$ this resonance function approaches

$\lim _{t \rightarrow \infty} T(\eta=0) \rightarrow 2 \pi t \delta\left(\beta_{n}\right)$,

yielding again diffusive behaviour, but in this case with the resonance function

$\mathcal{R}(\eta=0)=\lim _{t \rightarrow \infty} \frac{T(\eta=0)}{2 t}=\pi \delta\left(\beta_{n}\right)$.

This is fully consistent with Eq. (26), because of the same limit

$\lim _{\eta \rightarrow 0} \mathcal{R}(\boldsymbol{k})=\lim _{\eta \rightarrow 0} \frac{\eta}{\eta^{2}+\beta_{n}^{2}}=\pi \delta\left(\beta_{n}\right)$.

The use of the resonance function (30) is only problematic in cases where $\beta_{n}=k_{\|} v_{\|}+n \Omega=0$, as one encounters for $2 \mathrm{D}$ turbulence, see below (Sect. 4.2). In this case it is appropriate to go back to the general resonance function (24) which in the $\operatorname{limit} \beta_{n}=0$ is

$T\left(\beta_{n}=0\right) \rightarrow \frac{2 t}{\eta}\left[1-\frac{1-\mathrm{e}^{-\eta t}}{\eta t}\right]$.

For large times $\eta t \gg 1$ this resonance function approaches $T\left(\beta_{n}=0, \eta t \gg 1\right) \simeq 2 t / \eta$, indicating that the motion is still diffusive and that the resonance condition is

$\mathcal{R}\left(\beta_{n}=0\right)=\lim _{t \rightarrow \infty} \frac{T\left(\beta_{n}=0\right)}{2 t}=\frac{1}{\eta}=t_{\mathrm{c}}$, which agrees with the corresponding limit of Eq. (26). As an aside, we note that in the limit $\beta_{n}=0$ and $\eta=0$, the resonance functions (24) and (32) both imply superdiffusive behaviour,

$T\left(\beta_{n}=0, \eta=0\right)=t^{2}$.

We conclude, that in order to have diffusive behaviour of the Fokker-Planck coefficients in all limiting cases the existence of a finite correlation time of the magnetic fluctuations, i.e. condition (b) of Sect. 2.7, is essential.

\subsection{Fokker-Planck coefficients $D_{Y Y}$ and $D_{\perp}$}

By repeating the analysis for the equation of motion (2) we obtain in analogy to Eq. (27)

$$
\begin{aligned}
D_{Y Y}= & \lim _{t \rightarrow \infty} \frac{1}{2 t} \frac{1}{2 \pi} \int_{0}^{2 \pi} \mathrm{d} \phi_{0}\left\langle(\Delta Y)^{2}\right\rangle \\
= & \frac{v^{2}}{B_{0}^{2}} \sum_{n=-\infty}^{\infty} \int \mathrm{d}^{3} k \mathcal{R}(\boldsymbol{k}) \\
& \times\left\{\frac { 1 } { 4 } ( 1 - \mu ^ { 2 } ) P _ { \| \| ! } ^ { 0 } ( \boldsymbol { k } ) \left[J_{n+1}^{2}(W)+J_{n-1}^{2}(W)\right.\right. \\
& \left.-J_{n+1}(W) J_{n-1}(W)\left(\mathrm{e}^{2 \imath \psi}+\mathrm{e}^{-2 \imath \psi}\right)\right] \\
& +\frac{\mu^{2}}{2} J_{n}^{2}(W)\left(P_{R R}^{0}(\boldsymbol{k})-P_{R L}^{0}(\boldsymbol{k})-P_{L R}^{0}(\boldsymbol{k})+P_{L L}^{0}(\boldsymbol{k})\right) \\
& -\frac{\mu \sqrt{1-\mu^{2}}}{2 \sqrt{2}} J_{n}(W)\left[\left(P_{L \|}^{0}(\boldsymbol{k})-P_{R \|}^{0}(\boldsymbol{k})\right)\right. \\
& \times\left(J_{n+1}(W) \mathrm{e}^{-\imath \psi}-J_{n-1}(W) \mathrm{e}^{+\imath \psi}\right) \\
& +\left(P_{\| L}^{0}(\boldsymbol{k})-P_{\| R}^{0}(\boldsymbol{k})\right)\left(J_{n+1}(W) \mathrm{e}^{\imath \psi}\right. \\
& \left.\left.\left.-J_{n-1}(W) \mathrm{e}^{-\imath \psi}\right)\right]\right\} .
\end{aligned}
$$

For the sum of Fokker-Planck coefficients we then find

$$
\begin{aligned}
D_{\perp}(\mu)= & D_{X X}+D_{Y Y}=\frac{v^{2}}{2 B_{0}^{2}} \sum_{n=-\infty}^{\infty} \int \mathrm{d}^{3} k \mathcal{R}(\boldsymbol{k}) \\
& \times\left\{\left(1-\mu^{2}\right) P_{\|\| \|}^{0}(\boldsymbol{k})\left[J_{n+1}^{2}(W)+J_{n-1}^{2}(W)\right]\right. \\
& +2 \mu^{2} J_{n}^{2}(W)\left(P_{R R}^{0}(\boldsymbol{k})+P_{L L}^{0}(\boldsymbol{k})\right) \\
& -\mu \sqrt{2\left(1-\mu^{2}\right)} J_{n}(W)\left[P_{L \|}^{0}(\boldsymbol{k}) J_{n+1}(W) \mathrm{e}^{-\imath \psi}\right. \\
& +P_{R\|\|}^{0}(\boldsymbol{k}) J_{n-1}(W) \mathrm{e}^{\imath \psi} \\
& \left.\left.+P_{\| L}^{0}(\boldsymbol{k}) J_{n+1}(W) \mathrm{e}^{\imath \psi}+P_{\| R}^{0}(\boldsymbol{k}) J_{n-1}(W) \mathrm{e}^{-\imath \psi}\right]\right\}
\end{aligned}
$$

\section{Calculation of the Fokker-Planck coefficient $D_{\perp}$}

Now we use the general results for the perpendicular Fokker-Planck coefficient of the last Sect. (see Eq. (36)) to calculate $D_{\perp}$ for different turbulence geometries. To do this we have to specify the tensor $P_{l m}^{0}$. According to Matthaeus \& Smith (1981) the components of this tensor can be written as

$P_{l m}^{0}=g\left(k_{\perp}, k_{\|}\right) \cdot\left[\delta_{l m}-\frac{k_{l} k_{m}}{k^{2}}+i \sigma \epsilon_{l m n} \frac{k_{n}}{k}\right]$, 
with the magnetic helicity $\sigma$. The function $g\left(k_{\perp}, k_{\|}\right)$determines different turbulence geometries. We will consider two geometries explicitly in the following:

(a) slab turbulence, and

(b) pure 2D geometry.

With the results of these both geometries we are also able to calculate the perpendicular Fokker-Planck coefficient for composite geometry which is the subject of Sect. 3.3. For our calculations in both geometries we use the damping model of the dynamical magnetic turbulence.

\subsection{Fokker-Planck coefficient $D_{\perp}^{\text {slab }}$ for pure slab geometry}

In the case of pure slab geometry Eq. (37) becomes to

$P_{l m}^{0}=g\left(k_{\|}\right) \frac{\delta\left(k_{\perp}\right)}{k_{\perp}} \cdot\left[\delta_{l m}-\frac{k_{l} k_{m}}{k^{2}}+i \sigma \epsilon_{l m n} \frac{k_{n}}{k}\right]$.

Consequently, many components of the tensor $P_{l m}^{0}$ are zero except

$P_{R R}^{0}=(1-\sigma) g\left(k_{\|}\right) \frac{\delta\left(k_{\perp}\right)}{k_{\perp}}$,

$P_{L L}^{0}=(1+\sigma) g\left(k_{\|}\right) \frac{\delta\left(k_{\perp}\right)}{k_{\perp}}$.

Therefore we obtain for the perpendicular Fokker-Planck coefficient for slab geometry

$D_{\perp}^{\text {slab }}=\frac{v^{2} \mu^{2}}{B_{0}^{2}} \sum_{n=-\infty}^{+\infty} \int \mathrm{d}^{3} k \mathcal{R}_{D T}^{\mathrm{slab}} J_{n}^{2}(W)\left(P_{R R}^{0}+P_{L L}^{0}\right)$.

If we use the damping model of dynamical magnetic turbulence we have according to Eq. (26)

$\mathcal{R}_{D T}^{\text {slab }}=\frac{q_{\text {slab }}}{1+q_{\text {slab }}^{2}\left(k_{\|} v_{\|}+n \Omega\right)^{2}}$

because (Bieber et al. 1994)

$t_{\mathrm{c}}=\eta^{-1}=q_{\text {slab }}=\frac{1}{\alpha v_{A}\left|k_{\|}\right|}, \quad 0 \leq \alpha \leq 1$.

Using $J_{n}(0)=\delta_{n 0}$ we find for the Fokker-Planck coefficient

$D_{\perp}^{\text {slab }}=4 \pi \frac{\mu^{2} v^{2}}{B_{0}^{2}} \int_{-\infty}^{+\infty} \mathrm{d} k_{\|} g\left(\left|k_{\|}\right|\right) \frac{q_{\text {slab }}}{1+q_{\text {slab }}^{2} k_{\|}^{2} v_{\|}^{2}}$.

To proceed further, we have to specify the form of the power spectrum $g\left(\left|k_{\|}\right|\right)$. Here we use a power-law spectrum with a sharp cut-off at small wavenumbers:

$g\left(k_{\|}\right)=\left\{\begin{array}{cc}0 & \text { for } \quad\left|k_{\|}\right| \leq k_{\min } \\ g_{0}\left|k_{\|}\right|^{-s} & \text { for } \quad k_{\min } \leq\left|k_{\|}\right|\end{array}\right.$

with $1<s<2$. In Appendix $\mathrm{C}$ we discuss the results for a power spectrum with finite wave power at small wavennumbers (see Bieber et al. 1994; Teufel \& Schlickeiser 2003, SS03) which causes a divergence problem. It is convenient to express our results in terms of the following parameters:

$\epsilon=\frac{v_{A}}{v}$

$a=\frac{1}{\alpha \epsilon}=\frac{v}{\alpha v_{A}}=\frac{c}{\alpha v_{A}} \frac{r}{\sqrt{r_{0}^{2}+r^{2}}}$

$R_{L}=\frac{v}{\Omega}=\frac{p c}{B_{0}|q|}=\frac{r}{B_{0}}$

$R=R_{L} k_{\min }=r \frac{k_{\text {min }}}{B_{0}}$

with the cosmic ray particle's rigidity $r=\frac{p c}{|q|}$ and the constant $r_{0}=\frac{m c^{2}}{|q|}$. The Fokker-Planck coefficient (43) then becomes

$$
\begin{aligned}
D_{\perp}^{\text {slab }} & =\frac{8 \pi \alpha v_{A}}{B_{0}^{2}} \frac{a^{2} \mu^{2}}{1+a^{2} \mu^{2}} \int_{0}^{\infty} \frac{\mathrm{d} k_{\|}}{k_{\|}} g\left(k_{\|}\right) \\
& =\frac{8 \pi \alpha s v_{A}}{B_{0}^{2}} \cdot \frac{a^{2} \mu^{2}}{1+a^{2} \mu^{2}} \cdot g_{0} k_{\mathrm{min}}^{-s} .
\end{aligned}
$$

Expressing the constant $g_{0}$ in terms of the total fluctuating magnetic field strength of the slab component,

$$
\begin{aligned}
\left(\delta B_{\text {slab }}\right)^{2} & =\sum_{m=1}^{3}\left(\delta B_{m}\right)^{2} \\
& =\int \mathrm{d}^{3} k \int \mathrm{d}^{3} k^{\prime} \delta B_{m}(\boldsymbol{k}) \delta B_{m}^{*}\left(\boldsymbol{k}^{\prime}\right) \mathrm{e}^{i\left(\boldsymbol{k}-\boldsymbol{k}^{\prime}\right) \boldsymbol{x}} \\
& =\sum_{m=1}^{3} \int \mathrm{d}^{3} k P_{m m}(\boldsymbol{k})=8 \pi \int_{0}^{\infty} \mathrm{d} k_{\|} g\left(k_{\|}\right) \\
& =8 \pi g_{0} \int_{k_{\min }}^{\infty} \mathrm{d} k_{\|} k_{\|}^{-s} \\
& =\frac{8 \pi}{s-1} g_{0} k_{\min }^{1-s},
\end{aligned}
$$

yields

$D_{\perp}^{\text {slab }}=\frac{(s-1) \alpha v_{A}}{s k_{\min }}\left(\frac{\delta B_{\text {slab }}}{B_{0}}\right)^{2} \cdot \frac{a^{2} \mu^{2}}{1+a^{2} \mu^{2}}$.

\subsection{The Fokker-Planck coefficient for pure $2 D$ geometry $D_{\perp}^{2 \mathrm{D}}$}

For 2D geometry

$P_{l m}^{0}=g\left(k_{\perp}\right) \frac{\delta\left(k_{\|}\right)}{k_{\perp}} \cdot\left[\delta_{l m}-\frac{k_{l} k_{m}}{k^{2}}+i \sigma \epsilon_{l m n} \frac{k_{n}}{k}\right]$,

yielding for the individual non-zero components

$P_{R R}^{0}=P_{L L}^{0}=\frac{1}{2} g\left(k_{\perp}\right) \frac{\delta\left(k_{\|}\right)}{k_{\perp}}$

$P_{\mathrm{IIII}}^{0}=g\left(k_{\perp}\right) \frac{\delta\left(k_{\|}\right)}{k_{\perp}}$

$P_{\| R}^{0}=-P_{L \|}^{0}=\frac{\sigma}{\sqrt{2}} \mathrm{e}^{+i \Psi} \cdot g\left(k_{\perp}\right) \frac{\delta\left(k_{\|}\right)}{k_{\perp}}$

$P_{R \|}^{0}=-P_{\| L}^{0}=\frac{\sigma}{\sqrt{2}} \mathrm{e}^{-i \Psi} \cdot g\left(k_{\perp}\right) \frac{\delta\left(k_{\|}\right)}{k_{\perp}}$. 
With these components the Fokker-Planck coefficient (36) reduces to

$$
\begin{aligned}
D_{\perp}^{2 \mathrm{D}}= & \frac{v^{2}}{2 B_{0}^{2}} \sum_{n=-\infty}^{+\infty} \int \mathrm{d}^{3} k \mathcal{R}_{D T}^{2 \mathrm{D}} \cdot g\left(k_{\perp}\right) \frac{\delta\left(k_{\|}\right)}{k_{\perp}} \\
& \times\left[\left(1-\mu^{2}\right)\left(J_{n+1}^{2}(W)+J_{n-1}^{2}(W)\right)+2 \mu^{2} J_{n}^{2}(W)\right. \\
& \left.+2 \sigma \mu \sqrt{1-\mu^{2}} J_{n}(W)\left(J_{n+1}(W)-J_{n-1}(W)\right)\right] .
\end{aligned}
$$

For the calculations in the 2D-geometry we use

$t_{\mathrm{c}}=\eta^{-1}=q_{2 \mathrm{D}}=\frac{1}{\alpha v_{A} k_{\perp}}, \quad 0 \leq \alpha \leq 1$,

implying for the resonance function (21)

$\mathcal{R}_{D T}^{2 \mathrm{D}}=\frac{q_{2 \mathrm{D}}}{1+q_{2 \mathrm{D}}^{2}\left(k_{\|} v_{\|}+n \Omega\right)^{2}}$.

For vanishing magnetic helicity $\sigma=0$ the Fokker-Planck coefficient (51) then becomes

$$
\begin{aligned}
D_{\perp}^{2 \mathrm{D}}= & \frac{v^{2}}{2 B_{0}^{2}} \cdot \sum_{n=-\infty}^{+\infty} \int \mathrm{d}^{3} k \frac{q_{2 \mathrm{D}}}{1+q_{2 \mathrm{D}}^{2}\left(k_{\|} v_{\|}+n \Omega\right)^{2}} \\
& \times g\left(k_{\perp}\right) \frac{\delta\left(k_{\|}\right)}{k_{\perp}}\left[\left(1-\mu^{2}\right)\left(J_{n+1}^{2}(W)+J_{n-1}^{2}(W)\right)\right. \\
& \left.+2 \mu^{2} J_{n}^{2}(W)\right] .
\end{aligned}
$$

Two of the $k$-integrations can be readily performed, so that

$$
\begin{aligned}
D_{\perp}^{2 \mathrm{D}}= & \frac{2 \pi v^{2}}{\alpha v_{A} B_{0}^{2}} \sum_{n=-\infty}^{+\infty} \int_{0}^{\infty} \mathrm{d} k_{\perp} \frac{g\left(k_{\perp}\right)}{k_{\perp}} \frac{1}{1+\left(\frac{n \Omega}{\alpha v_{A} k_{\perp}}\right)^{2}} \\
& \times\left[\frac{\left(1-\mu^{2}\right)}{2}\left(J_{n+1}^{2}(W)+J_{n-1}^{2}(W)\right)+\mu^{2} J_{n}^{2}(W)\right],
\end{aligned}
$$

which can be written as

$D_{\perp}^{2 \mathrm{D}}(\sigma=0)=\frac{2 \pi \alpha v_{A} R_{L}^{2}}{B_{0}^{2}} \int_{0}^{\infty} \mathrm{d} k_{\perp} k_{\perp} g\left(k_{\perp}\right) f\left(k_{\perp}\right)$

where for brevity we introduced the functions

$f\left(k_{\perp}\right)=\left(1-\mu^{2}\right) G\left(k_{\perp}\right)+\mu^{2} H\left(k_{\perp}\right)$

$G\left(k_{\perp}\right)=\frac{1}{2} \sum_{n=-\infty}^{+\infty} \frac{J_{n-1}^{2}(W)+J_{n+1}^{2}(W)}{V^{2}+n^{2}}$

and

$H\left(k_{\perp}\right)=\sum_{n=-\infty}^{+\infty} \frac{J_{n}^{2}(W)}{V^{2}+n^{2}}$

with

$V=\frac{R_{L} k_{\perp}}{a}$,

(60) an

$W=R_{L} k_{\perp} u$ and $u=\sqrt{1-\mu^{2}}$. In Appendix A we derive the following approximations for the functions $G$ and $H$ :

$$
\begin{aligned}
G(V \ll 1, W \gg 1) & \approx \frac{J_{1}^{2}(W)}{V^{2}} \\
& \approx \frac{2}{\pi W V^{2}} \sin ^{2}\left(W-\frac{\pi}{4}\right) \approx \frac{1}{\pi W V^{2}} \\
G(V \ll 1, W \ll 1) & \approx 1+\frac{W^{2}}{4 V^{2}} \\
G(V \gg 1, V \ll W) & \approx \frac{1}{V W} \\
G(V \gg 1, V \gg W) & \approx \frac{1}{V^{2}}
\end{aligned}
$$

$$
\begin{aligned}
H(V \ll 1, W \gg 1) & \approx \frac{J_{0}^{2}(W)}{V^{2}} \\
& \approx \frac{2}{\pi W V^{2}} \cos ^{2}\left(W-\frac{\pi}{4}\right) \approx \frac{1}{\pi W V^{2}}
\end{aligned}
$$

$H(V \ll 1, W \ll 1) \approx \frac{1}{V^{2}}$

$H(V \gg 1, V \ll W) \approx \frac{1}{V W}$

$H(V \gg 1, V \gg W) \approx \frac{1}{V^{2}}$.

For a simple power law turbulence spectrum with sharp lowwavenumber cut-off as in Sect. 3.1, but now in $k_{\perp}$, we obtain for the Fokker-Planck coefficient (54)

$D_{\perp}^{2 \mathrm{D}}=\frac{2 \pi \alpha v_{A} R_{L}^{2}}{B_{0}^{2}} \cdot g_{0} \cdot k_{\min }^{1-s} \int_{k_{\min }}^{\infty} \mathrm{d} k\left(\frac{k}{k_{\min }}\right)^{1-s} f(k)$.

Again, the constant $g_{0}$ can be expressed in terms of the total fluctuating magnetic field strength in the 2D component:

$$
\begin{aligned}
\left(\delta B_{2 \mathrm{D}}\right)^{2} & =4 \pi \int_{0}^{\infty} \mathrm{d} k_{\perp} g\left(k_{\perp}\right) \\
& =4 \pi g_{0} \int_{k_{\min }}^{\infty} \mathrm{d} k_{\perp} k_{\perp}^{-s}=\frac{4 \pi}{s-1} g_{0} k_{\min }^{1-s}
\end{aligned}
$$

so that

$D_{\perp}^{2 \mathrm{D}}=\frac{(s-1) \alpha v_{A} R^{2}}{2 k_{\min }}\left(\frac{\delta B_{2 \mathrm{D}}}{B_{0}}\right)^{2} \cdot I_{\perp}^{2 \mathrm{D}}$

with

$I_{\perp}^{2 \mathrm{D}}=\int_{k_{\min }}^{\infty} \frac{\mathrm{d} k}{k_{\min }}\left(\frac{k}{k_{\min }}\right)^{1-s} \cdot f(k)$.

Substituting $x=k / k_{\min }$ we find

$I_{\perp}^{2 \mathrm{D}}=\int_{1}^{\infty} \mathrm{d} x x^{1-s} \cdot f(V, W)=\left(1-\mu^{2}\right) \cdot A+\mu^{2} \cdot B$

in terms of the integrals

$A=\int_{1}^{\infty} \mathrm{d} x x^{1-s} \cdot G(V, W)$

and

$B=\int_{1}^{\infty} \mathrm{d} x x^{1-s} \cdot H(V, W)$ 
Table 1. The different cases for the integrals $A$ and $B$.

\begin{tabular}{lll}
\hline \hline Case & $A$ & $B$ \\
\hline$a u \ll 1 \ll u R$ & $\frac{1}{s}\left(\frac{a}{R}\right)^{2}$ & $\frac{1}{s}\left(\frac{a}{R}\right)^{2}$ \\
$a u \ll u R \ll 1$ & $\frac{1}{s}\left(\frac{a}{R}\right)^{2}$ & $\frac{1}{s}\left(\frac{a}{R}\right)^{2}$ \\
$1 \ll a u \ll u R$ & $\frac{a}{s u R^{2}}$ & $\frac{a}{s u R^{2}}$ \\
$1 \ll u R \ll a u$ & $\frac{1}{(s+1) \pi} \frac{a^{2}}{u R^{3}}$ & $\frac{1}{(s+1) \pi} \frac{a^{2}}{u R^{3}}$ \\
$u R \ll a u \ll 1$ & $\frac{2}{s(2-s)}\left(\frac{a}{R}\right)^{2-s}$ & $\frac{1}{s}\left(\frac{a}{R}\right)^{2}$ \\
$u R \ll 1 \ll a u$ & $\frac{\pi(s+1)+4(2-s)}{4 \pi(2-s)(s+1)}\left(\frac{a}{R}\right)^{2}(u R)^{s}$ & $\frac{1}{s}\left(\frac{a}{R}\right)^{2}$ \\
\hline
\end{tabular}

In Eqs. (68)-(70) we use

$V=x \frac{R}{a}$

and

$W=x u R$.

We obtain for $G$ and $H$

$G(x \ll a / R, x \gg 1 / u R) \approx \frac{a^{2}}{\pi u R^{3} x^{3}}$

$G(x \ll a / R, x \ll 1 / u R) \approx 1+\frac{(a u)^{2}}{4}$

$G(x \gg a / R, 1 \ll a u) \approx \frac{a}{u R^{2} x^{2}}$

$G(x \gg a / R, 1 \gg a u) \approx \frac{a^{2}}{R^{2} x^{2}}$

$H(x \ll a / R, x \gg 1 / u R) \approx \frac{a^{2}}{\pi u R^{3} x^{3}}$

$H(x \ll a / R, x \ll 1 / u R) \approx \frac{a^{2}}{R^{2} x^{2}}$

$H(x \gg a / R, 1 \ll a u) \approx \frac{a}{u R^{2} x^{2}}$

$H(x \gg a / R, 1 \gg a u) \approx \frac{a^{2}}{R^{2} x^{2}}$.

Using Eqs. (73) and (74) to calculate the integrals $A$ and $B$ we obtain the approximations shown in Table 1.

\subsection{The Fokker-Planck coefficient for the composite slab/2D geometry $D_{\perp}^{X}$}

The Fokker-Planck coefficient in the composite slab/2D geometry is simply additive and can be written as

$D_{\perp}=D_{\perp}^{\text {slab }}+D_{\perp}^{2 \mathrm{D}}$.

If we replace $\delta B_{\text {slab }}$ in Eq. (48) and $\delta B_{2 \mathrm{D}}$ in Eq. (66) by the total turbulence $\delta B$ we find

$D_{\perp}^{\chi}=\chi D_{\perp}^{\text {slab }}+(1-\chi) D_{\perp}^{2 \mathrm{D}}$

where the parameter

$0 \leq \chi=\frac{\delta B_{\text {slab }}^{2}}{\delta B_{\text {slab }}^{2}+\delta B_{2 \mathrm{D}}^{2}} \leq 1$ measures the relative strength of slab turbulence with respect to the total turbulence $(\delta B)^{2}$.

With the results of the last subsections (Eqs. (48) and (66)) we immediately determine the Fokker-Planck coefficient in the composite model:

$D_{\perp}^{\chi}=\frac{(s-1) \alpha v_{A}}{s k_{\min }}\left(\frac{\delta B}{B_{0}}\right)^{2}\left\{\chi \frac{a^{2} \mu^{2}}{1+a^{2} \mu^{2}}+(1-\chi) \frac{R^{2}}{2} \cdot I_{\perp}^{2 \mathrm{D}}\right\}$

with $I_{\perp}^{2 \mathrm{D}}$ of Eq. (68).

\section{Perpendicular spatial diffusion coefficient and mean free path}

\subsection{Perpendicular spatial diffusion for slab geometry}

In this section we calculate the perpendicular spatial diffusion coefficient and the corresponding perpendicular mean free path for slab geometry. Using the perpendicular Fokker-Planck coefficient (48) in Eq. (4) we obtain

$$
\begin{aligned}
\kappa_{\perp}^{\text {slab }} & =\frac{(s-1) \alpha v_{A}}{s k_{\min }}\left(\frac{\delta B_{\text {slab }}}{B_{0}}\right)^{2} \int_{0}^{1} \mathrm{~d} \mu \frac{a^{2} \mu^{2}}{1+a^{2} \mu^{2}} \\
& =\frac{(s-1) \alpha v_{A}}{s k_{\min }}\left(\frac{\delta B_{\text {slab }}}{B_{0}}\right)^{2} \cdot\left(1-\frac{\arctan (a)}{a}\right)
\end{aligned}
$$

which can be approximated as

$$
\begin{aligned}
\kappa_{\perp}^{\text {slab }} \simeq & \frac{(s-1) \alpha v_{A}}{s k_{\min }}\left(\frac{\delta B_{\text {slab }}}{B_{0}}\right)^{2} \\
& \times \begin{cases}1 & \text { for } a \gg 1 \\
\frac{a^{2}}{3} \sim \frac{r^{2}}{r_{0}^{2}+r^{2}} & \text { for } a \ll 1 .\end{cases}
\end{aligned}
$$

For large values of $a \gg 1$ the spatial diffusion coefficient is independent of $a$ and therefore independent of the rigidity, whereas for small values $a \ll 1$ it increases $\propto r^{2}$ below $r \ll r_{0}$. The corresponding perpendicular mean free path is

$\lambda_{\perp}^{\text {slab }}=\frac{3 \kappa_{\perp}}{v}=\frac{3(s-1) \alpha v_{A}}{s k_{\min } v}\left(\frac{\delta B_{\text {slab }}}{B_{0}}\right)^{2}\left(1-\frac{\arctan (a)}{a}\right)$

with the approximative behaviour

$\lambda_{\perp}^{\text {slab }} \simeq \frac{3(s-1)}{s k_{\min }}\left(\frac{\delta B_{\text {slab }}}{B_{0}}\right)^{2} \begin{cases}a^{-1} & \text { for } a \gg 1 \\ \frac{a}{3} \sim \frac{r}{\sqrt{r_{0}^{2}+r^{2}}} & \text { for } a \ll 1 .\end{cases}$

For large values of $a \gg 1$ the mean free path decreases proportional to $\propto v^{-1}$, while at small values of $a \ll 1$ the mean free path increases $\propto v$. If we consider the case $\alpha \rightarrow 0$ we have $a \rightarrow \infty$ and we find that $\lambda_{\perp}^{\text {slab }}(\alpha \rightarrow 0) \rightarrow 0$.

\subsection{Perpendicular spatial diffusion for $2 D$ geometry}

Here we calculate the perpendicular spatial diffusion coefficient and the corresponding perpendicular mean free path in 2D geometry. We derive for the perpendicular spatial diffusion coefficient in 2D turbulence geometry

$\kappa_{\perp}^{2 \mathrm{D}}=\frac{(s-1) \alpha v_{A} R^{2}}{2 s k_{\min }} \cdot\left(\frac{\delta B_{2 \mathrm{D}}}{B_{0}}\right)^{2} \cdot K_{\perp}^{2 \mathrm{D}}$ 
Table 2. Analytical expressions for $K_{\perp, 1}^{2 \mathrm{D}}, K_{\perp, 2}^{2 \mathrm{D}}$ and $K_{\perp}^{2 \mathrm{D}}=K_{\perp, 1}^{2 \mathrm{D}}+K_{\perp, 2}^{2 \mathrm{D}}$. $\xi(s)$ denotes the function $\xi(s)=\left[\frac{1}{4(2-s)}+\frac{1}{\pi(s+1)}\right] \frac{\sqrt{\pi}}{4} \frac{\Gamma\left(\frac{s+2}{2}\right)}{\Gamma\left(\frac{s+5}{2}\right)}$.

\begin{tabular}{llll}
\hline \hline Case & $K_{\perp, 1}^{2 \mathrm{D}}$ & $K_{\perp, 2}^{2 \mathrm{D}}$ & $K_{\perp}^{2 \mathrm{D}}$ \\
\hline$a \ll R \ll 1$ & $\frac{2}{3 s}\left(\frac{a}{R}\right)^{2}$ & $\frac{1}{3 s}\left(\frac{a}{R}\right)^{2}$ & $\frac{1}{s}\left(\frac{a}{R}\right)^{2}$ \\
$a \ll 1 \ll R$ & $\frac{2}{3 s}\left(\frac{a}{R}\right)^{2}$ & $\frac{1}{3 s}\left(\frac{a}{R}\right)^{2}$ & $\frac{1}{s}\left(\frac{a}{R}\right)^{2}$ \\
$R \ll 1 \ll a$ & $(s+2) \xi(s)\left(\frac{a}{R}\right)^{2} R^{s}$ & $\frac{1}{3 s}\left(\frac{a}{R}\right)^{2}$ & $\frac{1}{3 s}\left(\frac{a}{R}\right)^{2}$ \\
$R \ll a \ll 1$ & $\frac{4}{3 s(2-s)}\left(\frac{a}{R}\right)^{2-s}$ & $\frac{1}{3 s}\left(\frac{a}{R}\right)^{2}$ & $\frac{1}{3 s}\left(\frac{a}{R}\right)^{2}$ \\
$1 \ll a \ll R$ & $\frac{\pi}{4 s} \frac{a}{R^{2}}$ & $\frac{\pi}{4} \frac{a}{R^{2}}$ & $\frac{\pi}{2 s} \frac{a}{R^{2}}$ \\
$1 \ll R \ll a$ & $\frac{1}{4(s+1)} \frac{a^{2}}{R^{3}}$ & $\frac{1}{4(s+1)} \frac{a^{2}}{R^{3}}$ & $\frac{1}{2(s+1)} \frac{a^{2}}{R^{3}}$ \\
\hline
\end{tabular}

with

$K_{\perp}^{2 \mathrm{D}}=\int_{0}^{1} \mathrm{~d} \mu I_{\perp}^{2 \mathrm{D}}=K_{\perp, 1}^{2 \mathrm{D}}+K_{\perp, 2}^{2 \mathrm{D}}$,

$K_{\perp, 1}^{2 \mathrm{D}}=\int_{0}^{1} \mathrm{~d} \mu\left(1-\mu^{2}\right) A(\mu)=\int_{0}^{1} \mathrm{~d} u \frac{u^{3}}{\sqrt{1-u^{2}}} A(u)$

and

$K_{\perp, 2}^{2 \mathrm{D}}=\int_{0}^{1} \mathrm{~d} \mu \mu^{2} B(\mu)=\int_{0}^{1} \mathrm{~d} u u \cdot \sqrt{1-u^{2}} B(u)$,

respectively. Table 2 shows the approximations for the last two integrals and for $K_{\perp}^{2 \mathrm{D}}$, yielding approximate formulas for $\kappa_{\perp}^{2 \mathrm{D}}$. With these the perpendicular mean free path of $2 \mathrm{D}$ geometry can be written as

$\lambda_{\perp}^{2 \mathrm{D}}=\frac{3(s-1)}{2 s} \frac{\alpha v_{A} R^{2}}{v k_{\min }} \cdot\left(\frac{\delta B_{2 \mathrm{D}}}{B_{0}}\right)^{2} \cdot K_{\perp}^{2 \mathrm{D}}$.

If we consider the case $\alpha \rightarrow 0$ we have $a \rightarrow \infty$. Table 2 then implies $\lambda_{\perp}^{2 \mathrm{D}}(\alpha \rightarrow 0) \sim 1 / \alpha \rightarrow \infty$, but, as shown in Sect. 2.8, in this formal limit the perpendicular cosmic ray transport is no longer diffusive.

\subsection{Composite slab/2D geometry}

For the case of composite geometry we can use Eqs. (80) and (83) to find

$$
\begin{aligned}
\kappa_{\perp}^{\chi}= & \chi \kappa_{\perp}^{\text {slab }}+(1-\chi) \kappa_{\perp}^{2 \mathrm{D}} \\
= & \frac{(s-1) \alpha v_{A}}{s k_{\min }}\left(\frac{\delta B}{B_{0}}\right)^{2} \cdot\left\{\chi \cdot\left(1-\frac{\arctan (a)}{a}\right)\right. \\
& \left.+(1-\chi) \cdot \frac{R^{2}}{2} \cdot K_{\perp}^{2 \mathrm{D}}\right\}
\end{aligned}
$$

with the total fluctuating magnetic field strength $\delta B^{2}=\delta B_{\text {slab }}^{2}+$ $\delta B_{2 \mathrm{D}}^{2}$. Although not necessary, mainly for illustrating our results we adopt the same value for the parameter $k_{\min }$ for the slab and the $2 \mathrm{D}$ contribution.

\section{Calculating $\kappa_{\perp}$ and $\lambda_{\perp}$ for heliospheric parameters}

Here we calculate $\kappa_{\perp}$ and $\lambda_{\perp}$ for electrons and protons for one set of typical heliospheric parameters and compare them with the analytical parallel diffusion results of SS03. For our calculations we use the same set of parameters appropriate for interplanetary conditions at $1 \mathrm{AU}$ as Bieber et al. (1994). For the magnetic background field we assume $B_{0}=4.12 \mathrm{nT}$ and for the Alfvén speed $v_{\mathrm{A}}=33.5 \mathrm{~km} \mathrm{~s}^{-1}$. For the both parameters of the power spectrum we used $k_{\min }=10^{-10} \mathrm{~m}^{-1}$ and $s=5 / 3$. The parameter $\alpha$ is assumed to be 1 .

In the following discussions we restrict the rigidity values to the interesting range $1 M V \ll r \ll 10^{4} M V$. With typical heliospheric parameters we then always have $R \ll 1 \ll a$.

\subsection{The perpendicular spatial diffusion coefficient $\kappa_{\perp}$}

The value of the spatial diffusion coefficient is given in terms of the constant $\kappa_{0}=10^{18} \mathrm{~cm}^{2} / \mathrm{s}$. For slab turbulence we then obtain

$$
\begin{aligned}
\frac{\kappa_{\perp}^{\text {slab }}}{\kappa_{0}} & =1.34 \cdot\left[1-\frac{\arctan a}{a}\right]\left(\frac{\delta B_{\text {slab }}}{B_{0}}\right)^{2} \\
& \approx 1.34 \cdot\left(\frac{\delta B_{\text {slab }}}{B_{0}}\right)^{2} .
\end{aligned}
$$

For the 2D coefficient we use Table 2 to obtain different approximations for $\kappa_{\perp}^{2 \mathrm{D}}$. In the case $R \ll 1 \ll a$ we find from Table 2:

$K^{2 \mathrm{D}}=\frac{1}{3 s}\left(\frac{a}{R}\right)^{2}$

and therefore for the perpendicular spatial diffusion coefficient

$\kappa_{\perp}^{2 \mathrm{D}}=\frac{(s-1)}{6 s} \frac{\alpha v_{\mathrm{A}}}{k_{\min }} \cdot a^{2} \cdot\left(\frac{\delta B_{2 \mathrm{D}}}{B_{0}}\right)^{2}$.

Using heliospheric parameters we obtain

$$
\begin{aligned}
\frac{\kappa_{\perp}^{2 \mathrm{D}}}{\kappa_{0}} & =0.223 \cdot a^{2} \cdot\left(\frac{\delta B_{2 \mathrm{D}}}{B_{0}}\right)^{2} \\
& =0.223 \cdot\left(\frac{c}{v_{\mathrm{A}}}\right)^{2} \cdot \frac{r^{2}}{r_{0}^{2}+r^{2}}\left(\frac{\delta B_{2 \mathrm{D}}}{B_{0}}\right)^{2} .
\end{aligned}
$$

The perpendicular spatial diffusion coefficient for protons and electrons from slab and 2D turbulence are shown in Fig. 1.

\subsection{The perpendicular mean free path $\lambda_{\perp}$}

The slab perpendicular mean free path becomes for heliospheric parameters

$$
\begin{aligned}
\lambda_{\perp}^{\text {slab }} & =0.08 \mathrm{AU} \cdot\left[\frac{1}{a}-\frac{\arctan a}{a^{2}}\right]\left(\frac{\delta B_{\text {slab }}}{B_{0}}\right)^{2} \\
& \approx 8.9 \times 10^{-6} \mathrm{AU} \frac{\sqrt{r_{0}^{2}+r^{2}}}{r}\left(\frac{\delta B_{\text {slab }}}{B_{0}}\right)^{2}
\end{aligned}
$$

where we used that $1 \mathrm{AU}=1.5 \times 10^{11} \mathrm{~m}$. For the $2 \mathrm{D}$ perpendicular mean free path we obtain under the restriction $R \ll 1 \ll a$ :

$$
\lambda_{\perp}^{2 \mathrm{D}}=\frac{(s-1)}{2 s} \frac{a}{k_{\min }} \cdot\left(\frac{\delta B_{2 \mathrm{D}}}{B_{0}}\right)^{2}
$$




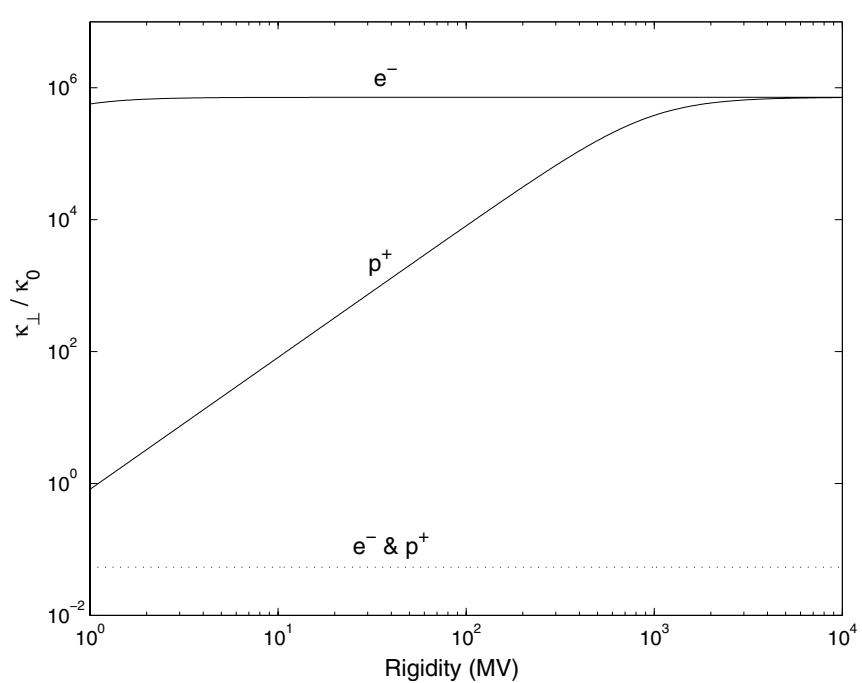

Fig. 1. Perpendicular spatial diffusion coefficient of 2D geometry (solid line) and slab geometry (dotted line) for protons $\left(\mathrm{p}^{+}\right)$and electrons $\left(\mathrm{e}^{-}\right)$for $\delta B / B_{0}=0.2$.

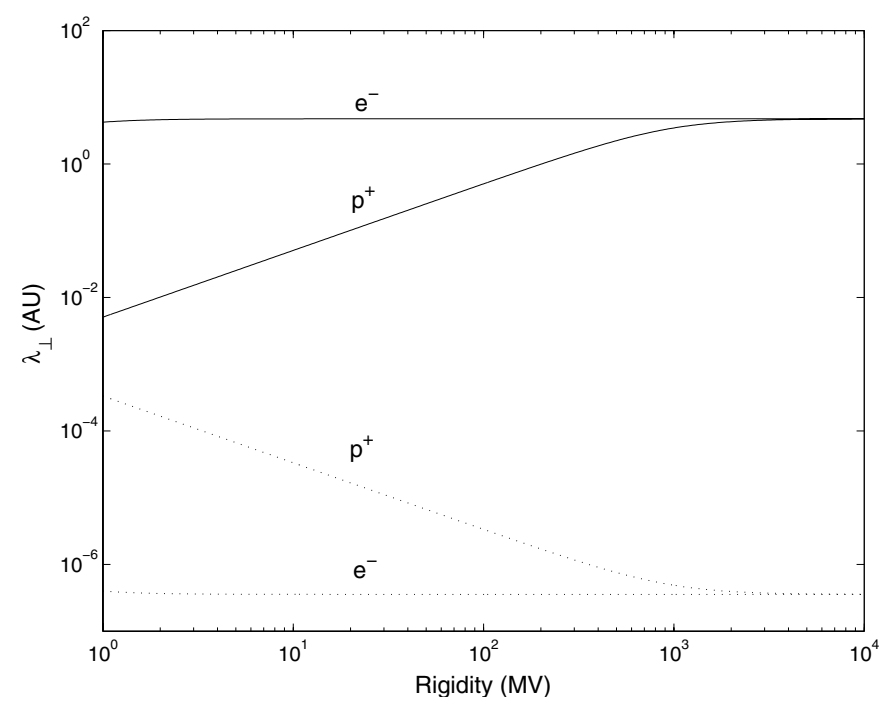

Fig. 2. Perpendicular mean free path of $2 \mathrm{D}$ geometry (solid line) and slab geometry (dotted line) for protons $\left(\mathrm{p}^{+}\right)$and electrons $\left(\mathrm{e}^{-}\right)$for $\delta B / B_{0}=0.2$.

which for heliospheric parameters yields

$$
\begin{aligned}
\lambda_{\perp}^{2 \mathrm{D}} & =0.0133 \mathrm{AU} \cdot a\left(\frac{\delta B_{2 \mathrm{D}}}{B_{0}}\right)^{2} \\
& =120 \mathrm{AU} \cdot \frac{r}{\sqrt{r_{0}^{2}+r^{2}}}\left(\frac{\delta B_{2 \mathrm{D}}}{B_{0}}\right)^{2} .
\end{aligned}
$$

The results for the perpendicular mean free path are shown in Fig. 2.

\subsection{The ratio $\lambda_{\perp} / \lambda_{\|}$}

In this subsection we calculate the ratio $\lambda_{\perp} / \lambda_{\|}$for slab, 2D and composite geometry. For $\lambda_{\|}^{2 \mathrm{D}}$ we can use the results of SS03 and for $\lambda_{\|}^{\text {slab }}$ the results of Teufel \& Schlickeiser (2003).
Together with Table 2 we obtain the following results:

$$
\begin{aligned}
& \lambda_{\perp}^{\text {slab }}=\frac{3(s-1)}{s a k_{\min }}\left(\frac{\delta B_{\text {slab }}}{B_{0}}\right)^{2} \\
& \lambda_{\perp}^{2 \mathrm{D}}=\frac{(s-1)}{2 s} \frac{a}{k_{\min }}\left(\frac{\delta B_{2 \mathrm{D}}}{B_{0}}\right)^{2} \\
& \lambda_{\|}^{\text {slab }}=\frac{6 s}{\pi(s-1)(2-s)(4-s)} \frac{R^{2-s}}{k_{\text {min }}}\left(\frac{B_{0}}{\delta B_{\text {slab }}}\right)^{2} \\
& \lambda_{\|}^{2 \mathrm{D}}=\frac{3 s \sqrt{\pi} 2^{s / 2}}{(s-1)(6-s)} \frac{\Gamma\left(\frac{8-s}{2}\right)}{\Gamma\left(\frac{7-s}{2}\right)} \frac{a R^{2-s}}{k_{\text {min }}}\left(\frac{B_{0}}{\delta B_{2 \mathrm{D}}}\right)^{2} .
\end{aligned}
$$

For the case $s=5 / 3$ these equations become to

$$
\begin{aligned}
& \lambda_{\perp}^{\text {slab }} \approx \frac{1.20}{k_{\min }} \frac{\alpha v_{\mathrm{A}}}{c} \frac{\sqrt{r_{0}^{2}+r^{2}}}{r}\left(\frac{\delta B_{\text {slab }}}{B_{0}}\right)^{2} \\
& \lambda_{\perp}^{2 \mathrm{D}} \approx \frac{0.2}{k_{\min }} \frac{c}{\alpha v_{\mathrm{A}}} \frac{r}{\sqrt{r_{0}^{2}+r^{2}}}\left(\frac{\delta B_{2 \mathrm{D}}}{B_{0}}\right)^{2} \\
& \lambda_{\|}^{\text {slab }} \approx \frac{6.14}{k_{\min }}\left(r \frac{k_{\mathrm{min}}}{B_{0}}\right)^{1 / 3}\left(\frac{B_{0}}{\delta B_{\text {slab }}}\right)^{2} \\
& \lambda_{\|}^{2 \mathrm{D}} \approx \frac{1.36}{k_{\min }} \frac{c}{\alpha v_{\mathrm{A}}} \frac{r}{\sqrt{r_{0}^{2}+r^{2}}}\left(r \frac{k_{\min }}{B_{0}}\right)^{1 / 3}\left(\frac{B_{0}}{\delta B_{2 \mathrm{D}}}\right)^{2} .
\end{aligned}
$$

With these results it is simple to calculate the ratio $\lambda_{\perp} / \lambda_{\|}$. If we do this we obtain for pure slab geometry

$\frac{\lambda_{\perp}^{\text {slab }}}{\lambda_{\|}^{\text {slab }}} \approx 5.04 \times 10^{-4} \frac{\sqrt{\left(\frac{r_{0}}{M V}\right)^{2}+\left(\frac{r}{M V}\right)^{2}}}{\left(\frac{r}{M V}\right)^{4 / 3}}\left(\frac{\delta B_{\text {slab }}}{B_{0}}\right)^{4}$

and for pure 2D geometry

$\frac{\lambda_{\perp}^{2 \mathrm{D}}}{\lambda_{\|}^{2 \mathrm{D}}} \approx 3.40 \cdot\left(\frac{r}{M V}\right)^{-1 / 3}\left(\frac{\delta B_{2 \mathrm{D}}}{B_{0}}\right)^{4}$.

These results can be seen in Fig. 3. In the case of composite geometry we have

$\lambda_{\perp}^{\chi}=\chi \cdot \lambda_{\perp}^{\text {slab }}+(1-\chi) \cdot \lambda_{\perp}^{2 \mathrm{D}}$.

If we assume that $\chi$ is not too small or too large we find that the slab component controlls the parallel mean free path (see SS03)

$\lambda_{\|}^{\chi} \approx \frac{1}{\chi} \cdot \lambda_{\|}^{\text {slab }}$

and the perpendicular mean free path is controlled by the $2 \mathrm{D}$ component

$\lambda_{\perp}^{\chi} \approx(1-\chi) \lambda_{\perp}^{2 \mathrm{D}}$.

Therefore the ratio of perpendicular and parallel mean free path can be written as

$\frac{\lambda_{\perp}^{\chi}}{\lambda_{\|}^{\chi}} \approx(1-\chi) \cdot \chi \cdot \frac{\lambda_{\perp}^{2 \mathrm{D}}}{\lambda_{\|}^{\text {slab }}}$. 


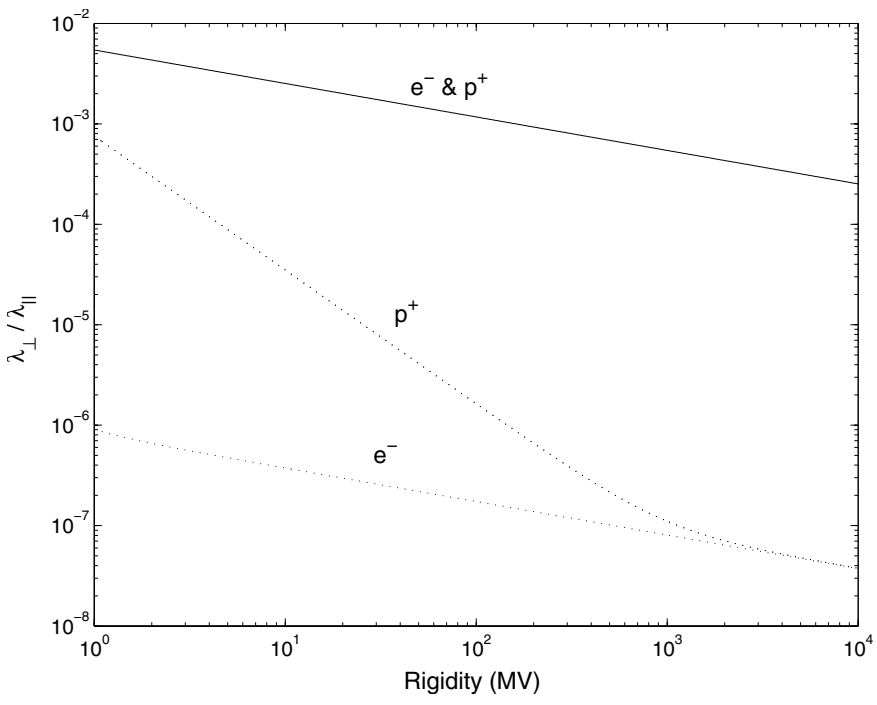

Fig. 3. The ratio $\lambda_{\perp} / \lambda_{\|}$for pure slab (dotted line) and pure $2 \mathrm{D}$ geometry (solid line) for protons $\left(\mathrm{p}^{+}\right)$and electrons $\left(\mathrm{e}^{-}\right)$for $\delta B / B_{0}=0.2$.

Note the symmetry of the mean free path ratio arround $\chi=0.5$ due to the factor $(1-\chi) \chi$ in Eq. (103). We find for heliospheric parameters

$$
\frac{\lambda_{\perp}^{\chi}}{\lambda_{\|}^{\chi}} \approx 6.74 \times 10^{3} \cdot(1-\chi) \cdot \chi \frac{(r / M V)^{2 / 3}}{\sqrt{\left(\frac{r_{0}}{M V}\right)^{2}+\left(\frac{r}{M V}\right)^{2}}}\left(\frac{\delta B}{B_{0}}\right)^{4} .
$$

Figure 4 shows the results of that ratio for electrons and Fig. 5 shows the results for protons. In both figures we calculated the ratio $\lambda_{\perp} / \lambda_{\|}$for different values of $\chi$ and for $\delta B / B_{0}=0.2$.

For non-relativistic particles we always find

$\frac{\lambda_{\perp}^{\chi}}{\lambda_{\|}^{\chi}}\left(r \ll r_{0}\right) \sim r^{2 / 3}$

and for relativistic particles

$\frac{\lambda_{\perp}^{\chi}}{\lambda_{\|}^{\chi}}\left(r \gg r_{0}\right) \sim r^{-1 / 3}$.

\subsection{Comparison with observations}

From proton observations we know that (see Palmer 1982) $0.02<\lambda_{\perp} / \lambda_{\|}<0.083$ over the rigidity range of $0.5 \mathrm{MV}<$ $r<5 G V$. The observations are not accurate enough to draw conclusions on the variation of the mean free path ratio with rigidity. By fitting the appropriate value of $\left(\frac{\delta B}{B_{0}}\right)^{4}$ the observed absolute values are in agreement with the quasilinear results for all three models: slab, 2D and composite turbulence. Future more precise observations especially of the rigidity variation of the ratio of mean free paths $\lambda_{\perp} / \lambda_{\|}$, both for protons and electrons, should allow a definite conclusion on the nature of interplanetary magnetic turbulence from the comparison with our theoretical predictions contained in Figs. 1-5.

\section{Summary and conclusions}

We have presented the quasilinear calculation of perpendicular diffusion of cosmic ray particles for weak dynamical magnetic

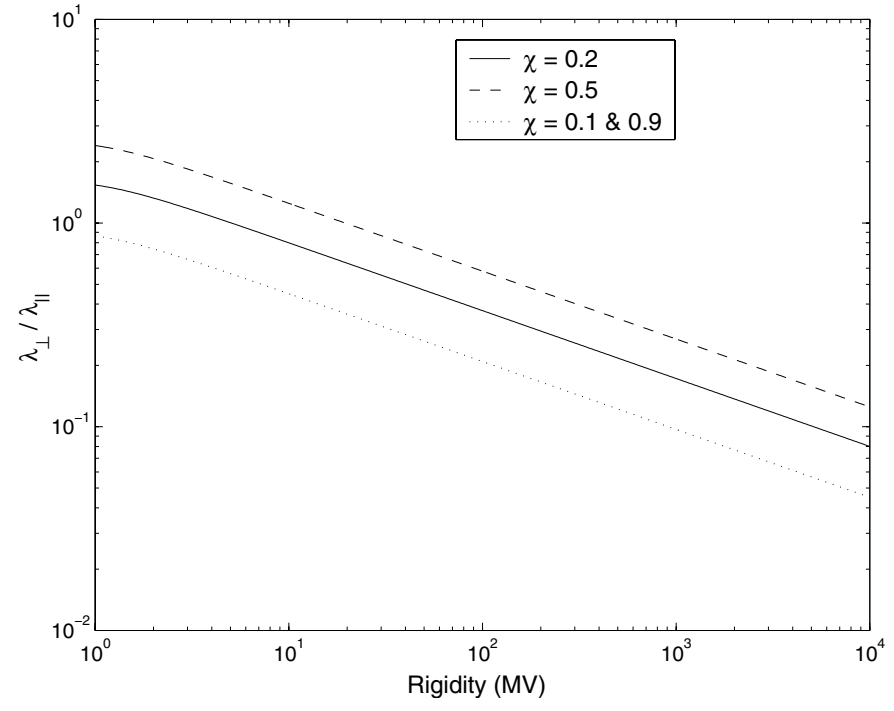

Fig. 4. The ratio $\lambda_{\perp} / \lambda_{\|}$as a function of the rigidity for electrons for different values of $\chi$ and for $\delta B / B_{0}=0.2$.

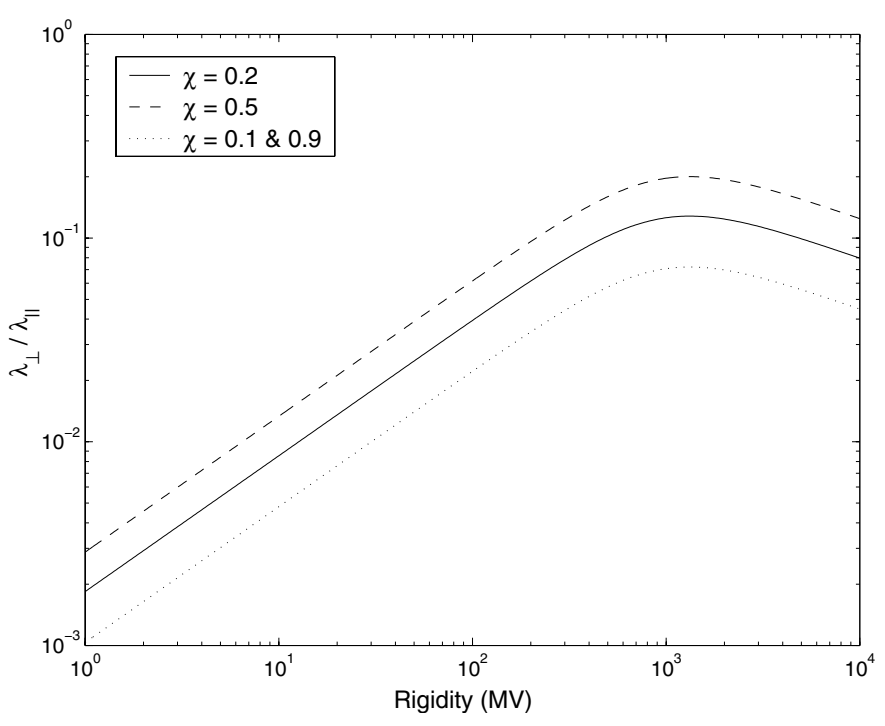

Fig. 5. The ratio $\lambda_{\perp} / \lambda_{\|}$as a function of the rigidity for protons for different values of $\chi$ and for $\delta B / B_{0}=0.2$.

turbulence of arbitrary geometry. Starting from the equations of motion we went point-by-point through the derivation of quasilinear Fokker-Planck coefficients, identifying seven necessary steps in this derivation. We demonstrated that, in order to have diffusive behaviour of the Fokker-Planck coefficients, the existence of a finite correlation time of the magnetic fluctuations is essential.

From the perpendicular Fokker-Planck coefficient $D_{\perp}$ we then deduced the perpendicular spatial diffusion coefficient $\kappa_{\perp}$ and the associated perpendicular mean free path $\lambda_{\perp}$ for the damping model of dynamical magnetic turbulence and three different turbulence geometries: slab, 2D turbulence and composite turbulence. For a Kolmogorov-type power spectrum we explicitly calculated these perpendicular transport parameters for electrons and protons for realistic heliospheric plasma 
parameters. The analytical form of the perpendicular diffusion coefficient is of great interest for studies of the solar modulation of galactic cosmic rays.

By comparing with our previous determination of the parallel transport parameters, we are able to predict the variation of the ratio of mean free paths $\lambda_{\perp} / \lambda_{\|}$with particle rigidity for the three turbulence models. The comparison of these predictiona with future accurate experimental determinations of the ratio of mean free paths will allow conclusions on the nature of interplanetary magnetic turbulence.

Acknowledgements. A.S. acknowledges support by the National Science Foundation under grant ATM-0000315. R.S. acknowledges support by the Deutsche Forschungsgemeinschaft through Sonderforschungsbereich 591. This work was completed while R.S. was a visiting professor at the University of California Riverside Institute of Geophysics and Planetary Physics. R.S. thanks Gary Zank, Director, for his kind hospitality and sponsorship through NSF grants ATM-0296113 and ATM-0317509.

\section{References}

Bieber, J. W., Matthaeus, W. H., Smith, C. W., et al. 1994, ApJ, 420, 294

Earl, J. A. 1974, ApJ, 193, 231

Giacalone, J., \& Jokipii, J. R. 1999, ApJ, 520, 201

Gradshteyn, I. S., \& Ryzhik, I. M. 1966 (New York: Academic Press)

Hall, D. E., \& Sturrock, P. A. 1968, Phys. Fluids, 10, 2620

Hasselmann, K., \& Wibberenz, G. 1968, Z. Geophys., 34, 353

Jaekel, U., \& Schlickeiser, R. 1992, J. Phys. G: Nucl. Part. Phys., 18, 1089

Le Roux, J. A., Zank, G. P., \& Ptuskin, V. S. 1999, JGR, 104, 24845

Mace, R. L., Matthaeus, W. H., \& Bieber, J. W. 2000, ApJ, 538, 192

Michalek, G. 2001, A\&A, 376, 667

Michalek, G., \& Ostrowski, M. 1998, A\&A, 337, 558

Palmer, I. D. 1982, Rev. Geophys. Space Phys., 20, 2, 335

Schlickeiser, R. 2002 (Berlin: Springer-Verlag)

Shalchi, A., \& Schlickeiser, R. 2004, ApJ, 604, 861 (SS03)

Teufel, A., \& Schlickeiser, R. 2003, A\&A, 397, 15

Zank, G. P., Matthaeus, W. H., Bieber, J. W., et al. 1998, JGR, 103, 2085 
A. Shalchi and R. Schlickeiser: Quasilinear perpendicular diffusion, Online Material p 1

\section{Online Material}


A. Shalchi and R. Schlickeiser: Quasilinear perpendicular diffusion, Online Material p 2

\section{Appendix A: Calculation of the series $G(x, y)$}

To calculate the Fokker-Planck coefficient in pure 2D geometry we have to calculate the series

$G(x, y)=\frac{1}{2} \sum_{n=-\infty}^{+\infty} \frac{J_{n-1}^{2}(x)+J_{n+1}^{2}(x)}{y^{2}+n^{2}}$.

It is not possible to solve this series analytically without using approximations. In this paper we use the same formalism to calculate the series as demonstrated in SS03. To start our calculations we write the sum above as

$G(x, y)=\frac{J_{1}^{2}(x)}{y^{2}}+\sum_{n=1}^{\infty} \frac{J_{n-1}^{2}(x)+J_{n+1}^{2}(x)}{y^{2}+n^{2}}$.

Now we use the both well known formulas (see Gradshteyn \& Ryzhik 1966):

$$
\begin{aligned}
J_{n-1}^{2}(x)= & -\frac{2}{\pi}(-1)^{n} \int_{0}^{\pi / 2} \mathrm{~d} \Theta J_{0}(2 x \cos \Theta) \\
& \times \cos (2 \Theta n-2 \Theta) \\
= & -\frac{2}{\pi}(-1)^{n} \int_{0}^{\pi / 2} \mathrm{~d} \Theta J_{0}(2 x \cos \Theta) \\
& \times[\cos (2 \Theta n) \cos (2 \Theta)+\sin (2 \Theta n) \sin (2 \Theta)]
\end{aligned}
$$

and

$$
\begin{aligned}
J_{n+1}^{2}(x)= & -\frac{2}{\pi}(-1)^{n} \int_{0}^{\pi / 2} \mathrm{~d} \Theta J_{0}(2 x \cos \Theta) \\
& \times \cos (2 \Theta n+2 \Theta) \\
= & -\frac{2}{\pi}(-1)^{n} \int_{0}^{\pi / 2} \mathrm{~d} \Theta J_{0}(2 x \cos \Theta) \\
& \times[\cos (2 \Theta n) \cos (2 \Theta)-\sin (2 \Theta n) \sin (2 \Theta)]
\end{aligned}
$$

to get

$$
\begin{aligned}
J_{n-1}^{2}(x) & +J_{n+1}^{2}(x)=-\frac{4}{\pi}(-1)^{n} \\
& \times \int_{0}^{\pi / 2} \mathrm{~d} \Theta J_{0}(2 x \cos \Theta) \cos (2 \Theta n) \cos (2 \Theta) .
\end{aligned}
$$

Therefore we obtain

$$
\begin{aligned}
G(x, y)= & \frac{J_{1}^{2}(x)}{y^{2}}-\frac{4}{\pi} \int_{0}^{\pi / 2} \mathrm{~d} \Theta J_{0}(2 x \cos \Theta) \cos (2 \Theta) \\
& \times \sum_{n=1}^{\infty} \frac{(-1)^{n} \cos (2 \Theta n)}{n^{2}+y^{2}} .
\end{aligned}
$$

Now we can calculate the sum using (Gradshteyn \& Ryzhik 1966)

$\sum_{n=1}^{\infty} \frac{(-1)^{n}}{n^{2}+y^{2}} \cos (2 \Theta n)=\frac{\pi}{2 y} \frac{\cosh (2 \Theta y)}{\sinh (\pi y)}-\frac{1}{2 y^{2}}$

and we find

$$
\begin{aligned}
G(x, y)= & \frac{J_{1}^{2}(x)}{y^{2}}+\frac{2}{\pi y^{2}} \int_{0}^{\pi / 2} \mathrm{~d} \Theta J_{0}(2 x \cos \Theta) \cos (2 \Theta) \\
& -\frac{2}{y \sinh (\pi y)} \int_{0}^{\pi / 2} \mathrm{~d} \Theta J_{0}(2 x \cos \Theta) \\
& \times \cos (2 \Theta) \cosh (2 \Theta y) .
\end{aligned}
$$

Using

$\int_{0}^{\pi / 2} \mathrm{~d} \Theta J_{0}(2 x \cos \Theta) \cos (2 \Theta)=-\frac{\pi}{2} J_{1}^{2}(x)$

the series $G$ can be finally written as

$$
\begin{aligned}
G(x, y)= & -\frac{2}{y \sinh (\pi y)} \int_{0}^{\pi / 2} \mathrm{~d} \Theta J_{0}(2 x \cos \Theta) \\
& \times \cos (2 \Theta) \cosh (2 \Theta y) .
\end{aligned}
$$

This result is still exact, but to proceed with our calculations we must consider special cases for $x$ and $y$.

\section{The case $y \ll 1, x \gg 1$}

In this case we can consider Eq. (A.10) for small $y$ to obtain

$$
\begin{aligned}
G(x, y) & =-\frac{2}{\pi y^{2}} \int_{0}^{\pi / 2} \mathrm{~d} \Theta J_{0}(2 x \cos \Theta) \cos (2 \Theta) \\
& =\frac{J_{1}^{2}(x)}{y^{2}}
\end{aligned}
$$

\section{The case $y \ll 1, x \ll 1$}

In this case we must develop the functions in Eq. (A.10) up to the next order:

$J_{0}(2 x \cos \Theta) \approx 1-x^{2} \cos ^{2} \Theta$,

$\cosh (2 \Theta y) \approx 1+2 \Theta^{2} y^{2}$,

$\frac{1}{\sinh (\pi y)} \approx \frac{1}{\pi y}\left(1-\frac{\pi^{2} y^{2}}{6}\right)$

with these approximations it is simple to calculate $G$ for small arguments

$$
\begin{aligned}
G(x, y) \approx & -\frac{4}{\pi} \cdot \int_{0}^{\pi / 2} \mathrm{~d} \Theta \Theta^{2} \cdot \cos (2 \Theta) \\
& +\frac{2 x^{2}}{\pi y^{2}} \cdot \int_{0}^{\pi / 2} \mathrm{~d} \Theta \cos (2 \Theta) \cdot \cos ^{2} \Theta
\end{aligned}
$$

which can be written as

$G(x, y) \approx 1+\frac{x^{2}}{4 y^{2}}$.

\section{The case $y \gg 1$}

In this case Eq. (A.10) can be written as

$$
\begin{aligned}
G(x, y)= & -\frac{2}{y} \int_{0}^{\pi / 2} \mathrm{~d} \Theta J_{0}(2 x \cos \Theta) \cos (2 \Theta) \\
& \times\left[\mathrm{e}^{(2 \Theta-\pi) y}+\mathrm{e}^{-(2 \Theta+\pi) y}\right] .
\end{aligned}
$$

With the integral transformation $z=\pi-2 \Theta$ we find

$$
\begin{aligned}
G(x, y)= & \frac{1}{y} \int_{0}^{\pi} \mathrm{d} z J_{0}\left(2 x \sin \left(\frac{z}{2}\right)\right) \cos (z) \\
& \times\left[\mathrm{e}^{-z y}+\mathrm{e}^{-(2 \pi-z) y}\right] .
\end{aligned}
$$

Now we consider large $y$ to approximate the integral. If $y$ is a

large number the contribution to the integral comes from very 
small values of $z$ because of the exponential function. Therefore we can expand the upper limit of the integral to infinity and we can approximate the circular functions to obtain:

$G(x, y)=\frac{1}{y} \int_{0}^{\infty} \mathrm{d} z J_{0}(x z) \mathrm{e}^{-y z}$.

The integral is elementary and yields

$G(x, y)=\frac{1}{y} \frac{1}{\sqrt{x^{2}+y^{2}}}$.

We finally find the both cases:

$G(y \gg 1, y \ll x)=\frac{1}{x y}$
$G(y \gg 1, y \gg x)=\frac{1}{y^{2}}$.

Finally all the cases can be written as:

$$
\begin{aligned}
G(y \ll 1, x \gg 1) & \approx \frac{J_{1}^{2}(x)}{y^{2}} \approx \frac{2}{\pi x y^{2}} \sin ^{2}(x-\pi / 4) \\
& \approx \frac{1}{\pi x y^{2}}
\end{aligned}
$$

$G(y \ll 1, x \ll 1) \approx 1+\frac{x^{2}}{4 y^{2}}$

$G(y \gg 1, y \ll x) \approx \frac{1}{x y}$

$G(y \gg 1, y \gg x) \approx \frac{1}{y^{2}}$.

With these approximations it is possible to calculate the perpendicular Fokker-Planck coefficient for special cases. We also calculated the series $G(x, y)$ numerically to test Eq. (A.20). We found that the agreement is accurate for all cases.

\section{Appendix B: Calculation of the series $H(x, y)$}

For calculating the Fokker-Planck coefficient in pure 2D geometry we also have to calculate the series

$H(x, y)=\sum_{n=-\infty}^{+\infty} \frac{J_{n}^{2}(x)}{y^{2}+n^{2}}$.

In this section we use the same method to calculate the series as in the section before. To start our calculations we write the sum above as

$H(x, y)=\frac{J_{0}^{2}(x)}{y^{2}}+2 \sum_{n=1}^{\infty} \frac{J_{n}^{2}(x)}{y^{2}+n^{2}}$.

With the well known integral representation for Bessel functions

$J_{n}^{2}(x)=\frac{2}{\pi}(-1)^{n} \int_{0}^{\pi / 2} \mathrm{~d} \Theta J_{0}(2 x \cos \Theta) \cos (2 \Theta n)$

we can rewrite the series as

$$
\begin{aligned}
H(x, y)= & \frac{J_{0}^{2}(x)}{y^{2}}+\frac{4}{\pi} \int_{0}^{\pi / 2} \mathrm{~d} \Theta J_{0}(2 x \cos \Theta) \\
& \times \sum_{n=1}^{\infty} \frac{(-1)^{n}}{n^{2}+y^{2}} \cos (2 \Theta n) .
\end{aligned}
$$

Using again Eq. (A.7) we get

$$
H(x, y)=\frac{2}{y \sinh (\pi y)} \int_{0}^{\pi / 2} \mathrm{~d} \Theta J_{0}(2 x \cos \Theta) \cosh (2 \Theta y)
$$

where we also used

$\frac{2}{\pi} \int_{0}^{\pi / 2} \mathrm{~d} \Theta J_{0}(2 x \cos \Theta)=J_{0}^{2}(x)$.

Now we must consider special cases for $x$ and $y$ to simplify Eq. (B.5).

\section{The case $y \ll 1$}

In this case we can use

$\cosh (2 \Theta y) \approx 1$

and Eq. (B.6) to find

$H(x, y \ll 1) \approx \frac{J_{0}^{2}(x)}{y^{2}}$.

If $x \ll 1$ and $y \ll 1$ we obtain

$H(x \ll 1, y \ll 1) \approx \frac{1}{y^{2}}$.

\section{The case $y \gg 1$}

In the case of large $y$ Eq. (B.5) can be written as

$$
\begin{aligned}
H(x, y) & \approx \frac{1}{y} \int_{0}^{\pi} \mathrm{d} z J_{0}\left(2 x \sin \left(\frac{z}{2}\right)\right) \cdot\left[\mathrm{e}^{-z y}+\mathrm{e}^{-(2 \pi-z) y}\right] \\
& \approx \frac{1}{y} \int_{0}^{\infty} \mathrm{d} z J_{0}(x z) \mathrm{e}^{-z y} \\
& \approx \frac{1}{y} \frac{1}{\sqrt{x^{2}+y^{2}}} .
\end{aligned}
$$

For deriving this equation we used the same approximations as in deriving Eq. (A.17). If we collect all the results we finally find:

$$
\begin{aligned}
& H(y \ll 1, x \gg 1) \approx \frac{J_{0}^{2}(x)}{y^{2}} \approx \frac{2}{\pi x y^{2}} \cos ^{2}(x-\pi / 4) \approx \frac{1}{\pi x y^{2}} \\
& H(y \ll 1, x \ll 1) \approx \frac{1}{y^{2}} \\
& H(y \gg 1, y \ll x) \approx \frac{1}{x y} \\
& H(y \gg 1, y \gg x) \approx \frac{1}{y^{2}} .
\end{aligned}
$$

With these approximations it is possible to calculate the perpendicular Fokker-Planck coefficient for special cases. We also calculated the series $H(x, y)$ numerically to test Eq. (B.11). We found that the agreement is accurate for all cases.

\section{Appendix C: A power spectrum with finite wave power at small wavenumbers}

In this section we discuss the results for a power spectrum with finite wave power at small wavenumbers. To do this we 
consider pure slab- and pure 2D-geometry for the damping model of dynamical magnetic turbulence:

\section{C.1. The case of pure slab geometry}

According to Eq. (46) the Fokker-Planck coefficient can be written as

$D_{\perp}^{\text {slab }}=\frac{8 \pi \alpha v_{\mathrm{A}}}{B_{0}^{2}} \frac{a^{2} \mu^{2}}{1+a^{2} \mu^{2}} \int_{0}^{\infty} \frac{\mathrm{d} k_{\|}}{k_{\|}} g\left(k_{\|}\right)$.

Now we assume a power spectrum with finite wave power at small wavenumbers:

$g\left(k_{\|}\right)=\left\{\begin{array}{clc}g_{0} k_{\min }^{-s} & \text { for } & k_{\|} \leq k_{\min } \\ g_{0} k_{\|}^{-s} & \text { for } & k_{\min } \leq k_{\|}\end{array}\right.$

to obtain

$$
\begin{aligned}
D_{\perp}^{\text {slab }} & \sim \int_{0}^{\infty} \frac{\mathrm{d} k_{\|}}{k_{\|}} g\left(k_{\|}\right) \\
& =g_{0} k_{\min }^{s} \int_{0}^{k_{\min }} \frac{\mathrm{d} k_{\|}}{k_{\|}}+g_{0} \int_{k_{\min }}^{\infty} \frac{\mathrm{d} k_{\|}}{k_{\|}} k_{\|}^{-s}
\end{aligned}
$$

and we find

$D_{\perp}^{\text {slab }} \sim \lim _{k_{0} \rightarrow 0} \int_{k_{0}}^{\infty} \frac{\mathrm{d} k_{\|}}{k_{\|}}=\lim _{k_{0} \rightarrow 0} \ln \left(\frac{k_{\min }}{k_{0}}\right) \rightarrow \infty$.

Note: this is a feature of the damping model of dynamical magnetic turbulence. If we consider the case of $\alpha \rightarrow 0$, often refered as magnetostatic limit (see Bieber et al. 1994) we must go back to Eq. (40):

$D_{\perp}^{\text {slab }}=4 \pi \frac{v^{2} \mu^{2}}{B_{0}^{2}} \int_{-\infty}^{+\infty} \mathrm{d} k_{\|} \mathcal{R}_{0}^{\mathrm{slab}}(n=0) g\left(k_{\|}\right)$.

Now we use

$\mathcal{R}_{0}^{\text {slab }}=\pi \delta\left(k_{\|} v_{\|}+n \Omega\right)$

and therefore

$\mathcal{R}_{0}^{\text {slab }}(n=0)=\pi \delta\left(k_{\|} v_{\|}\right)=\frac{\pi}{v|\mu|} \delta\left(k_{\|}\right)$

to obtain

$$
\begin{aligned}
D_{\perp}^{\text {slab }} & =4 \pi^{2} \frac{v^{2} \mu^{2}}{B_{0}^{2}} \frac{1}{v|\mu|} g(0) \\
& =\frac{(s-1) \pi}{2} v|\mu| k_{\min }^{-1}\left(\frac{\delta B}{B_{0}}\right)^{2}
\end{aligned}
$$

With this result we can calculate the perpendicular mean free path and we find

$\lambda_{\perp}=\frac{3}{4}(s-1) \pi k_{\min }^{-1} \cdot\left(\frac{\delta B}{B_{0}}\right)^{2}$

which is similar to the results derived by Le Roux et al. (1999) and Zank et al. (1998).

\section{C.2. The case of pure $2 D$ geometry}

The 2D-Fokker-Planck coefficient can be written as (see Eq. (51))

$D_{\perp}^{2 \mathrm{D}}=\frac{\pi v^{2}}{B_{0}^{2}} \sum_{n=-\infty}^{+\infty} \int_{0}^{\infty} \mathrm{d} k_{\perp} Q_{n}\left(k_{\perp}\right)$

with

$Q_{n}\left(k_{\perp}\right)=\mathcal{R}_{D T}^{2 \mathrm{D}} g\left(k_{\perp}\right)\left[\left(1-\mu^{2}\right)\left(J_{n+1}^{2}(W)+J_{n-1}^{2}(W)\right)\right.$

$$
\left.+2 \mu^{2} J_{n}^{2}(W)\right]
$$

if we assume a vanishing magnetic helicity. Now we restrict our analysis to the $n=0$ contribution and we find

$Q_{0}\left(k_{\perp} \rightarrow 0\right) \sim \frac{g\left(k_{\perp}\right)}{k_{\perp}} \sim \frac{1}{k_{\perp}}$

and therefore

$D_{\perp}^{2 \mathrm{D}} \sim \lim _{k_{0} \rightarrow 0} \int_{k_{0}}^{\infty} \frac{\mathrm{d} k_{\perp}}{k_{\perp}}=\lim _{k_{0} \rightarrow 0} \ln \left(\frac{k_{\min }}{k_{0}}\right) \rightarrow \infty$

if we use the damping model of dynamical magnetic turbulence and the same power spectrum as in Eq. (C.2) but now for $k_{\perp}$. Note: for slab- and for 2D-geometry the perpendicular FokkerPlanck coefficient goes to infinity if we assume a power spectrum with finite wave power at small wavenumbers. 